\title{
Cortically Driven Immediate-Early Gene Expression Reflects Modular Influence of Sensorimotor Cortex on Identified Striatal Neurons in the Squirrel Monkey
}

\author{
H.B. Parthasarathy and A.M. Graybiel \\ Department of Brain and Cognitive Sciences, Massachusetts Institute of Technology, Cambridge, Massachusetts 02139
}

Current understanding of basal ganglia function emphasizes their involvement in the focal, context-dependent release of motor and cognitive circuits in the brainstem and frontal lobes. How such selective action can arise despite the existence of massively convergent inputs from the cerebral cortex is unknown. However, anatomical work has suggested that specificity could be achieved in corticostriatal circuits by modular patterns of convergent and divergent cortical inputs to striatal projection neurons. To test for such modular activation of striatal neurons, we electrically microstimulated physiologically identified sites in the primary somatosensory (SI) and primary motor (MI) cortex of the squirrel monkey. We compared the efferent fiber distributions anterogradely traced from these sites to the distributions of striatal neurons activated by microstimulation to express Fos- and Jun B-like immediate-early gene proteins. We show that the microstimulation of sensorimotor cortex induces Fos and Jun B expression in localized cell clusters in the putamen and that these clusters match the anatomical input fiber clusters (matrisomes). The modular activation of striatal neurons by sensorimotor cortex seems likely. Unexpectedly, $>75 \%$ of the Fos-positive nuclei in densely labeled cell clusters were in enkephalin-immunoreactive neurons. This expression pattern suggests that the primate sensorimotor cortex exerts a differential influence on the enkephalin- ergic (indirect pathway) as opposed to the substance $\mathrm{P} /$ dynorphin (direct pathway) projection neurons of the putamen. The densely labeled clusters of Fos-labeled enkephalinergic neurons occurred within larger zones containing sparsely distributed Fos-labeled parvalbumin neurons. Moreover, when the cortical stimulation induced expression of Fos-like protein only in sparsely distributed neurons, almost every putamenal neuron expressing Fos was a parvalbumin-containing (GABAergic) interneuron. These patterns suggest a model in which the primate sensorimotor cortex can target parvalbumincontaining inhibitory interneurons, which in turn depress the remaining neuronal activity within and around matrisomes in a feed-forward manner until sufficient coherent cortical input can overcome the inhibition to influence selectively enkephalinergic projection neurons in the activated matrisomes. Tuning of cortical input by striatal interneurons thus may be an important mechanism by which broader anatomical connections are dynamically adjusted to achieve selective flow of information through the basal ganglia.

Key words: basal ganglia; striatum; somatosensory cortex; motor cortex; Fos; Jun B; immediate-early gene; interneuron; parvalbumin; coherent activity; primate; tract tracing; striosome-matrix
A single medium spiny projection neuron in the striatum may receive $>10,000$ excitatory inputs from the cerebral cortex. Any single cortical fiber, however, may synapse only once or a few times on any such projection neuron (Wilson, 1995). How, then, are cortical inputs coordinated to bring about activation of striatal neurons? One idea that has been raised is that convergence of coherently active cortical input fibers is necessary to activate striatal neurons and that such convergence is brought about by the modular organization of corticostriatal inputs terminating in striosomes or in patchy matrix domains called matrisomes. Selective convergence and divergence of corticostriatal inputs from different cortical areas thus could serve to delimit functional modules in

Received Sept. 30, 1996; revised Jan. 7, 1997; accepted Jan. 9, 1997.

This work was supported by National Institutes of Health Javits Award 5 R01NS25529 and by the Stanley Foundation. H.B.P. was supported by National Institute of Mental Health Training Grant T32MH15761. We thank Mr. H. F. Hall, who was responsible for the photography; Ms. D. Major and Mr. G. Holm for help with histology and immunohistochemistry; and Dr. S. Berretta for the design of Figure $9 C$. We also thank Dr. R. Bravo for his gift of Jun B antiserum and Dr. C. Cavada for consultation on the use of BDA.

Correspondence should be addressed to Dr. Ann M. Graybiel, Department of Brain and Cognitive Sciences, Massachusetts Institute of Technology, E25-618, Cambridge, MA 02139.

Copyright (C) 1997 Society for Neuroscience 0270-6474/97/172477-15\$05.00/0 the striatum, providing a substrate for differential contextdependent activation of striatal projection neurons (Flaherty and Graybiel, 1991, 1993a; Parthasarathy et al., 1992; Cowan and Wilson, 1994; Graybiel et al., 1994; Plenz and Aertsen, 1994; Graybiel and Kimura, 1995; Houk and Wise, 1995).

In the monkey, overlapping and nonoverlapping clusters of corticostriatal fibers and striatal projection neurons have indeed been demonstrated neuroanatomically in tract-tracing studies (Flaherty and Graybiel, 1993a). The functional consequences of such anatomical configurations, however, have yet to be discovered. In the work described here, we attempted to measure the functional influence of cortical inputs on their target domains in the squirrel monkey striatum. We combined anterograde corticostriatal tract tracing from electrophysiologically identified sites in the somatosensory and motor cortex with the immunohistochemical detection of immediate-early gene (IEG) proteins expressed in response to focal electrical stimulation of these cortical sites. The IEG proteins we chose to monitor, Fos and Jun B, are leucine zipper transcription factors that are rapidly and transiently expressed in response to a variety of cellular signals (Sheng and Greenberg, 1990). In the rodent, electrical stimulation of the cerebral cortex induces the expression of Fos and Jun B in striatal 
Table 1. Summary of experimental protocols

\begin{tabular}{|c|c|c|c|c|c|c|c|c|c|}
\hline Case & $\begin{array}{l}\text { Electrode } 1 \\
\text { Location }\end{array}$ & $\begin{array}{l}\text { Electrode } 2 \\
\text { Location }\end{array}$ & $\begin{array}{l}\text { Distance } \\
\text { between } \\
\text { electrodes }\end{array}$ & $\begin{array}{l}\text { Current } \\
\text { intensity }\end{array}$ & $\begin{array}{l}\text { Electrode } \\
\text { type }\end{array}$ & $\begin{array}{l}\text { Stim } \\
\text { posto } \\
(\mathrm{min})\end{array}$ & $\begin{array}{l}\text { duration/ } \\
\text { survival }\end{array}$ & $\begin{array}{l}\text { Tracer } \\
\text { type }\end{array}$ & $\begin{array}{l}\text { Tracer } \\
\text { location }\end{array}$ \\
\hline SMRC43 & MI foot & MI foot & $1.5 \mathrm{~mm} \mathrm{AP}$ & $500 \mu \mathrm{A}$ & Platinum-iridium & 60 & 120 & {$\left[{ }^{35} \mathrm{~S}\right] \mathrm{methionine}$} & SI 3b shoulder \\
\hline SMRC44 & MI forearm & MI forearm & $1.5 \mathrm{~mm} \mathrm{ML}$ & $500 \mu \mathrm{A}$ & Platinum-iridium & 60 & 120 & BDA & $\mathrm{SI} 3 \mathrm{~b} / 1$ forearm \\
\hline SMRC46L & $\begin{array}{c}\text { SI 3b foot } \\
\text { D5-D3 }\end{array}$ & SI 1 foot & $2.0 \mathrm{~mm} \mathrm{ML}$ & $\begin{array}{l}500 \mu \mathrm{A} \\
\quad(10 \mathrm{~min}) \\
\text { then } 350 \mu \mathrm{A}\end{array}$ & Tungsten & 115 & 120 & {$\left[{ }^{35} \mathrm{~S}\right]$ methionine } & SI $3 b$ foot \\
\hline SMRC46R & MI hand & MI hand & $\ll 1.0 \mathrm{~mm} \mathrm{ML}$ & $500-200 \mu \mathrm{A}$ & Tungsten & 115 & 135 & None & \\
\hline SMRC48L & SI lower leg & & & $350 \mu \mathrm{A}$ & $\begin{array}{c}\text { Concentric } \\
\text { bipolar }\end{array}$ & 90 & 120 & None & \\
\hline SMRC48R & SI $3 b$ foot & SI 3b foot & $1.7 \mathrm{~mm} \mathrm{AP}$ & $350 \mu \mathrm{A}$ & Tungsten & 90 & 120 & {$\left[{ }^{35} \mathrm{~S}\right]$ methionine } & SI 3b foot \\
\hline SMRC49 & SI 3b hand D5 & SI 1 hand D4/D5 & $2.3 \mathrm{~mm} \mathrm{AP}$ & $350 \mu \mathrm{A}$ & Tungsten & 90 & 120 & $\begin{array}{l}{\left[{ }^{35} \mathrm{~S}\right] \text { methionine }} \\
\text { BDA } \\
\text { WGA-HRP }\end{array}$ & $\begin{array}{l}\text { SI } 3 b / 1 \text { hand } \\
\text { SI } 1 \text { hand } \\
\text { SI } 3 b / 1 \text { forearm }\end{array}$ \\
\hline SMRC51 & MI hand/wrist & MI hand & $2.0 \mathrm{~mm} \mathrm{ML}$ & $350 \mu \mathrm{A}$ & Tungsten & 90 & 120 & & \\
\hline
\end{tabular}

neurons, presumably by evoking the release of glutamate at corticostriatal synapses (Fu and Beckstead, 1992; Wan et al., 1992; Besson et al., 1993; Parthasarathy et al., 1994; Liste et al., 1995).

With these combined techniques, we were able to detect matrisomes as the patchy distribution domains of somatotopically defined sensorimotor cortical input fibers within the matrix compartment of the putamen and, in the same or in adjacent sections, ensembles of postsynaptic striatal neurons sufficiently stimulated to express immunodetectable Fos or Jun B. In addition, by double-label immunohistochemistry, we were able to determine the phenotypes of the responsive striatal neurons. Our findings suggest the presence of dynamic focusing mechanisms in the primate striatum by which the sensorimotor cortex can activate highly selective subsets of striatal neurons in modular ensembles.

\section{MATERIALS AND METHODS}

Experiments were performed on eight hemispheres in six squirrel monkeys (Saimiri sciureus and Saimiri sciureus macrodon), typically in two- or three-stage surgeries.

Before the first two surgical procedures, monkeys were tranquilized with ketamine $(12 \mathrm{mg} / \mathrm{kg})$; anesthetized with xylazine $(0.7 \mathrm{mg} / \mathrm{kg})$; and given atropine $(0.04 \mathrm{mg} / \mathrm{kg})$, dexamethasone $(0.1 \mathrm{mg} / \mathrm{kg})$, and DiTrim $(30$ $\mathrm{mg} / \mathrm{kg}$ ). Anesthesia was maintained with ketamine (in doses of $5 \mathrm{mg} / \mathrm{kg}$ ) and was supplemented, before surgical procedures, by buprenorphine $(0.01 \mathrm{mg} / \mathrm{kg})$. Lactated Ringer's solution $(2.3 \mathrm{ml} / \mathrm{kg} / \mathrm{hr}$, usually intravenously) was given during the experiment. Between surgeries, the monkeys were treated with Tribrissen $(30 \mathrm{mg} / \mathrm{kg}$, b.i.d., p.o.).

During the first surgery, selected regions of the somatosensory and motor cortex were exposed under sterile surgical conditions, and a thin layer of viscasil was placed over the exposed neocortex. Primary motor cortex (MI) was located by eliciting visually monitored movements with intracortical microstimulation. Trains of symmetric biphasic paired pulses (cathodal pulse leading, $0.2 \mathrm{msec}$ each, 250 pulse pairs/sec) or single cathodal pulses $(0.2 \mathrm{msec}$ pulse width, $250 \mathrm{pulses} / \mathrm{sec})$ were delivered for $50-100$ msec through a low-impedance platinum-iridium electrode inserted into the cortex. Movements were typically evoked at stimulus intensities of $\sim 50 \mu \mathrm{A}$. Areas $3 \mathrm{a}, 3 \mathrm{~b}$, and 1 of primary somatosensory cortex (SI) was located by monitoring neural activity recorded during tactile stimulation $(\sim 1 \mathrm{M} \Omega$ platinum-iridium electrodes, MicroProbe, Clarksburg, MD). Sites of electrode penetrations were marked on an enlarged photograph of the exposed cortex. Because of the multiple, closely spaced surgeries required for these experiments, just enough time was taken to identify the somatotopic representation within the cortical areas of interest based on previous experience in this laboratory of mapping SI and MI in the squirrel monkey (Flaherty and Graybiel, 1991, 1993a, 1995). The average spacing of stimulation and recording sites was $0.5 \mathrm{~mm}$. After the completion of mapping, anterograde tracers were introduced into the cortex through a glass micropipette at the site chosen during the mapping session and were identified by reference to the photographic print on which electrode penetrations were marked. The micropipettes were attached to a pressure-injection system (Picopump, World Precision Instruments, Gaithersburg, MD) capable of delivering tracer in aliquots as small as 1-2 $\mathrm{nl}$. In four monkeys, $\left[{ }^{35} \mathrm{~S}\right]$ methionine $(10-70 \mathrm{nl}, 200 \mu \mathrm{Ci} / \mu \mathrm{l}, \mathrm{NEN})$ and wheat germ agglutinin-conjugated horseradish peroxidase (WGA-HRP, 15-30 nl, 10\%) were pressureinjected into electrophysiologically determined sites in MI or SI. In two monkeys, biotinylated dextran amine (BDA; 80-200 $\mathrm{nl}, 10 \%$ ) was pressure-injected into a single chosen site in MI or SI. Injected amounts were monitored by tracking a fiducial air bubble in the pump line, and injection site loci were recorded on the photographic mapping record. After tracer injection, the surgical field was thoroughly lavaged with sterile saline, neomycin-sulfate/dexamethasone ophthalmic solution was applied to the surface of the cortex, the cortex was covered with gelfoam, and the opening was closed in layers.

In the monkeys in which BDA was injected, a second surgery was performed $10 \mathrm{~d}$ later. The original surgical field was reexposed, and SI and MI cortex was mapped further. $\left[{ }^{35}\right.$ S]methionine or WGA-HRP was then pressure-injected into a second, electrophysiologically determined location.

Parameters for stimulation to induce IEG expression were derived from a preliminary series of experiments performed in rats (Parthasarathy, 1996). In these experiments, monopolar stimulation proved much less effective than bipolar stimulation at comparable intensities for inducing striatal IEGs. For electrode distances $<1.5 \mathrm{~mm}$, area and density of striatal IEG induction was quite variable and proportional to the separation distance between electrodes.

Two days after the last tracer injections were made, a surgery was performed to permit microstimulation of the sensorimotor cortex. Each monkey was anesthetized with Nembutal $(20 \mathrm{mg} / \mathrm{kg}$, i.p. $)$, and the surgical field was again exposed. Parallel bipolar platinum-iridium (MicroProbe) or tungsten (A-M Systems) wire electrodes, $\sim 1.5-2 \mathrm{~mm}$ apart, were used to stimulate the cortex for $1-2 \mathrm{hr}$ at sites chosen from the previous mapping experiments and identified by vascular landmarks and, in the case of MI stimulation, by microstimulation. Stimulation was delivered in symmetric biphasic pulses of $0.2 \mathrm{msec}$ pulse width each, at 200-500 $\mu \mathrm{A}$, in $40 \mathrm{msec}$ trains (4 trains $/ \mathrm{sec}, 250 \mathrm{pulse} / \mathrm{sec}$; see Table 1). For the experiments in which sites in MI were stimulated, the stimulus intensity was adjusted so that a discrete, well-localized movement of the somatotopically corresponding body part was visible throughout most of the stimulation period. Depth of anesthesia was judged by monitoring muscle tone and heart rate and, if necessary, supplemental Nembutal ( 5-7 $\mathrm{mg} / \mathrm{kg}$ ) was given intraperitoneally during the stimulation period. Supplements were given to three of the monkeys (SMRC46, 48, and 49). Two hours after the onset of stimulation, the monkey was deeply anesthetized with Nembutal (on average, $50 \mathrm{mg} / \mathrm{kg}$ ) and was perfused transcardially with $4 \%$ paraformaldehyde in $0.1 \mathrm{M}$ phosphate buffer containing $0.9 \%$ saline (PBS) or $4 \%$ paraformaldehyde in $0.1 \mathrm{~m}$ sodium cacodylate buffer.

Tissue preparation and histology. Brains were blocked transversely or in the midsagittal plane, and the blocks, after being soaked in buffer con- 
taining $20-30 \%$ sucrose or glycerol, were cut on a freezing microtome into $40-$ or $20-\mu \mathrm{m}$-thick coronal or sagittal sections. Sections were processed in serially adjacent sets for WGA-HRP histochemistry, autoradiography, BDA histochemistry, or IEG protein immunohistochemistry as appropriate.

For autoradiography, sections were mounted on chrome-alum-subbed slides, dried, defatted, dipped in Kodak NTB-2 emulsion, dried again, and stored in light-tight boxes at $-20^{\circ} \mathrm{C}$ for $1-8$ weeks. Sections were developed in Kodak D19 developer and counterstained through the emulsion. WGA-HRP histochemistry was performed by a modified TMB method (Mesulam, 1978). BDA histochemistry was identical to the avidin-biotin incubation for immunohistochemistry.

Immunohistochemistry to detect IEGs was performed with commercially available polyclonal antiserum raised against c-Fos (Oncogene Science Ab-2, 1:200 and 1:500) or with Jun B antiserum (1:3000 lot \#725-5 and 1:10,000 lot \#725/3) kindly donated by Dr. R. Bravo (BristolMyers Squibb, Princeton, NJ). The patterns of immunoreactivity observed with the polyclonal antisera used refer strictly to antigens detected with these antisera and may involve related proteins or peptide species, including Fos-like and Jun B-like proteins. Such terms as Jun B-positive, Jun B-like immunoreactive, and Jun B-immunoreactivity are used accordingly. Commercially available antibodies and antisera were used to detect ChAT (Incstar, 1:2000), parvalbumin (Sigma, St. Louis, MO; 1:1000), calretinin (Swant, 1:5000) or met-enkephalin (Incstar, 1:1000). Immunohistochemical detection of dynorphin was attempted without success with commercially available antisera (Peninsula Dyn A(1-8) and Dyn B, 1:1000, 1:5000, 1:10,000) and rat dynorphin B 1-29 antiserum donated by Dr. S. Watson.

Sections processed for immunohistochemistry were first treated with $10 \%$ methanol and $3 \%$ hydrogen peroxide to inhibit endogenous peroxidase activity and to eliminate cross-reaction with WGA-HRP, then were incubated with $5 \%$ normal secondary serum and incubated overnight at $4^{\circ} \mathrm{C}$ in primary antiserum in PBS containing $0.2 \%$ Triton X-100. After a 1-2 $\mathrm{hr}$ incubation with biotinylated secondary antibody, sections were processed with avidin-biotin kits (Vector Laboratories, Burlingame CA) and nickel-enhanced diaminobenzidine (DAB). Dual-antigen immunohistochemistry was performed serially. Sections were washed overnight in PBS-Triton X-100; carried through the first antiserum incubation; passed through blocking steps in hydrogen peroxide, avidin, and biotin; and finally were incubated with the second antiserum. BSA was substituted throughout for normal secondary serum. The first antigen was detected with nickel-enhanced DAB (black reaction product), the second with cacodylate-buffered DAB (brown reaction product). In instances in which double-labeling of Fos and enkephalin antigens was performed, the Fos-like immunoreactivity was demonstrated with gold-conjugated secondary antiserum (1:50) followed by silver intensification as necessary with Intense-M kit (Amersham, Arlington Heights, IL). To obtain double-labeling for Fos-like or Jun B-like immunoreactivity and NADPH diaphorase (nitric oxide synthase), NADPH diaphorase histochemistry (Vincent et al., 1983) was performed after the immunohistochemical (DAB) reaction for Fos-like or Jun B-like immunoreactivity.

Data analysis. Sections were charted by camera lucida, either by hand or with a Eutectics computerized image analysis system. Fos-positive and Jun B-positive nuclei were charted at $20 \times$. Unless otherwise specified, the transcription factor plots are schematic representations of the observed distributions (i.e., within the densest fields of induction, not every nucleus was counted). For purposes of comparison only, the approximate density of Fos induction was calculated by counting the nuclei on three crosssections and dividing the total counts by the volume in which they were found (area of the induction $\times 40 \mu \mathrm{m}$ ).

For comparison of the distributions of fibers labeled with $\left[{ }^{35} \mathrm{~S}\right] \mathrm{methi}-$ onine or WGA-HRP with the distributions of striatal neurons expressing Fos-like or Jun B-like immunoreactivity, serial sections were plotted, unless otherwise indicated. The outline of the section processed for IEG protein immunohistochemistry was first traced on the Eutectics system and printed. Blood vessels were then traced onto the printed image by hand with the aid of a Wild microscope with drawing-tube attachment. The adjacent section processed for autoradiography was aligned according to the blood vessel pattern, and the outlines of the corticostriatal fiber projection were drawn. This final composite drawing was retraced on a Summagraphics tablet into the Eutectics system computer and saved as a Hewlett Packard Graphics Language (HPGL) file. Plots of Fos- or Jun B-positive nuclei within the boundaries of the same region of the putamen were also saved as an HPGL file, and the two images were converted and aligned with an automatic function of Corel-Draw 3.0 (Corel Corporation).

Doubly labeled neurons were charted at either $40 \times$ or $63 \times$ in sets of sections spaced $\sim 1 \mathrm{~mm}$ apart. The study was restricted to Fosimmunoreactive neurons stained with markers for neuronal phenotypes. For each section, a border around the region of gene induction in the putamen just sufficient to enclose all Fos-positive nuclei was drawn at low power. For interneuronal stains, all singly and doubly labeled interneurons within the region of gene induction were counted, and the numbers of doubly labeled neurons were expressed as percentages of all interneurons. For staining of Fos-positive neurons and enkephalinergic neurons, $40 \times$ microscope fields were chosen randomly within the region of dense gene induction and all Fos-positive nuclei were plotted. The numbers of doubly labeled Fos-positive, enkephalin-positive neurons within these field were expressed as percentages of the total Fos-positive population.

BDA allows detection of individual fibers and putative terminal varicosities (Brandt and Apkarian, 1992; Veenman et al., 1992; Rajakumar et al., 1993; Reiner et al., 1993). In BDA-stained sections, labeled varicosities were plotted and counted at $63 \times$. Sections were typically $40 \mu \mathrm{m}$ thick. A plane of focus showing the apparent maximal density of varicosities was chosen, and all varicosities were charted within this plane. BDA-labeled and Fos-positive nuclei were stained on the same sections and thus could be charted coordinately. The charts were converted to HPGL files and then to Corel-Draw files.

\section{RESULTS}

\section{IEG induction in the putamen in response to stimulation of sensorimotor cortex}

We electrically stimulated sites in either SI or MI cortex in eight hemispheres to induce the expression of IEG proteins (Table 1). We chose stimulation conditions as described in a preliminary report on this technique in the rat (Besson et al., 1993), which we also tested in the rat (Parthasarathy et al., 1994). Trains of high-frequency pulses (250 pulse pairs/sec in $40 \mathrm{msec}$ trains, 4 trains/sec) were delivered for 1-2 hr through parallel bipolar electrodes spaced 1.5-2 $\mathrm{mm}$ apart. In all cases for which we followed these parameters, Fos-like immunoreactivity and Jun B-like immunoreactivity were induced in neurons in the ipsilateral putamen. In two cases, one in which stimuli were applied through a single concentric bipolar electrode (SMRC48L), and one in which parallel bipolar electrodes were spaced much less than 1 $\mathrm{mm}$ apart (SMRC46R), little or no specific IEG induction was observed. We found similar patterns of induction for stimulation of MI and SI. In two unstimulated control hemispheres, no Fos-positive nuclei were observed in the putamen. Apparently, constitutive expression of Jun B-like immunoreactivity was present in scattered neurons throughout the striatum in untreated control hemispheres.

\section{Comparisons of afferent fiber distributions and IEG induction stimulated from somatosensory cortex}

Cases could be divided into two groups according to the density of induction within these zones: weakly activated ( $<4000$ nuclei/ $\mathrm{mm}^{3}$; SMRC43, SMRC48R, SMRC49, SMRC51) and strongly activated $\left(>12,000\right.$ nuclei $/ \mathrm{mm}^{3}$; SMRC44, SMRC46L). In both groups, Fos and Jun B were expressed in variably patchy domains. The zones of Fos and Jun B induction in the putamen ranged in cross-sectional area from 0.3 to $1.5 \mathrm{~mm}$. In the cases with strong induction, clear focal clusters of Fos-positive and Jun B-positive nuclei were present in addition to a more scattered distribution of immunoreactive nuclei (Fig. 1). The distributions of Fos-positive and Jun B-positive nuclei lay in corresponding regions of the putamen, but the Jun B-immunoreactive neurons were more densely distributed in the clusters, and it was necessary to judge the pattern of induction against a background of weak constitutive expression. 

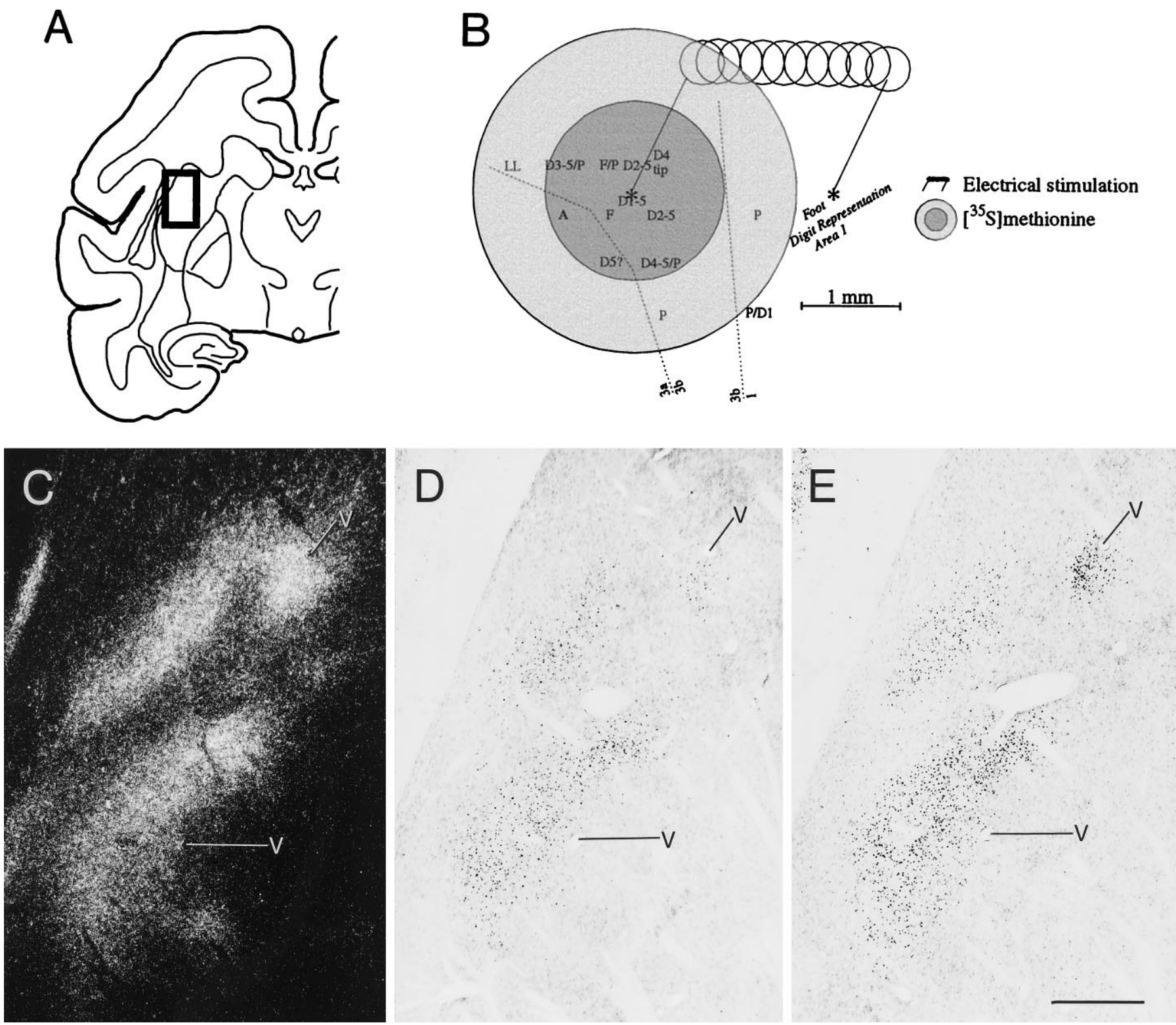

Figure 1. Electrical stimulation of the cutaneous foot digit representation in area SI induces Fos in multiple patches in the primate striatum that correspond to the afferent patches labeled by injection of $\left[{ }^{35} \mathrm{~S}\right]$ methionine in the $3 \mathrm{~b}$ foot digit representation. Case SMRC46L. $A$, Region shown at higher magnification in $C-E$. $B$, Map of sites in cortical area SI identified by neuronal recording. $A$, Ankle; $D$, digit; $F$, foot; $L L$, lower leg; $P$, foot pad. Dotted lines indicate the approximate borders between areas $3 \mathrm{a}$ and $3 \mathrm{~b}$ and areas $3 \mathrm{~b}$ and 1 . Darker circle indicates the reconstructed $\left[{ }^{35} \mathrm{~S}\right] \mathrm{methionine}$ injection site. The lighter concentric circle indicates the less intensely labeled marginal zone of the injection site. Stimulation sites shown at asterisks indicate positions of bipolar electrodes. Stimulation was applied across areas $3 \mathrm{~b}$ and 1 . The fields of afferent fibers labeled from the injection site and IEG expression induced by the electrical stimulation in the rectangular region outlined are shown in $C-E$ in high-magnification photomicrographs of serially adjacent transverse sections. $C$, Patches of $\left[{ }^{35}\right.$ S $]$ methionine-labeled corticostriatal fibers viewed under dark-field illumination. $D$, Patches of Fos-positive nuclei. $E$, Patches of Jun B-positive nuclei. $V$, Blood vessels used as fiducial points. Scale bar, $0.5 \mathrm{~mm}$.

To meet the condition of having parallel bipolar stimulating electrodes $\sim 1.5-2 \mathrm{~mm}$ apart, a distance near or exceeding the width of subareas within SI-MI cortex, we usually used the strategy of placing the parallel bipolar stimulating electrodes across homologous body part representations in adjacent areas (e.g., across areas 3a and $3 \mathrm{~b}$ of SI cortex). Such homologous sites in SI and MI cortex have convergent corticostriatal projections to tightly overlapping matrisomes within the ipsilateral putamen in the squirrel monkey (Flaherty and Graybiel, 1991, 1995).

In three hemispheres, we combined electrical stimulation of the
SI cortex with injections of anterograde tracers in somatotopically corresponding sites in SI and compared the subsequent expression of Fos and Jun B proteins with the distributions of labeled corticostriatal fibers.

\section{IEG induction and afferent labeling from somatotopically corresponding sites in SI cortex}

In one hemisphere (SMRC46L, Fig. 1), we mapped the foot region of area SI and injected $\left[{ }^{35} \mathrm{~S}\right]$ methionine into the digit representation of area $3 \mathrm{~b}$. We matched the injection site by electrically stimulating across the cutaneous digit representation 


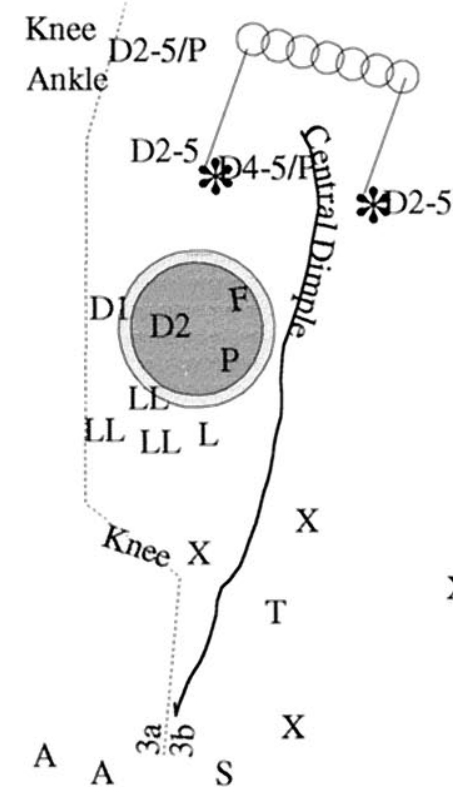

$\mathrm{S}$

Occ

A

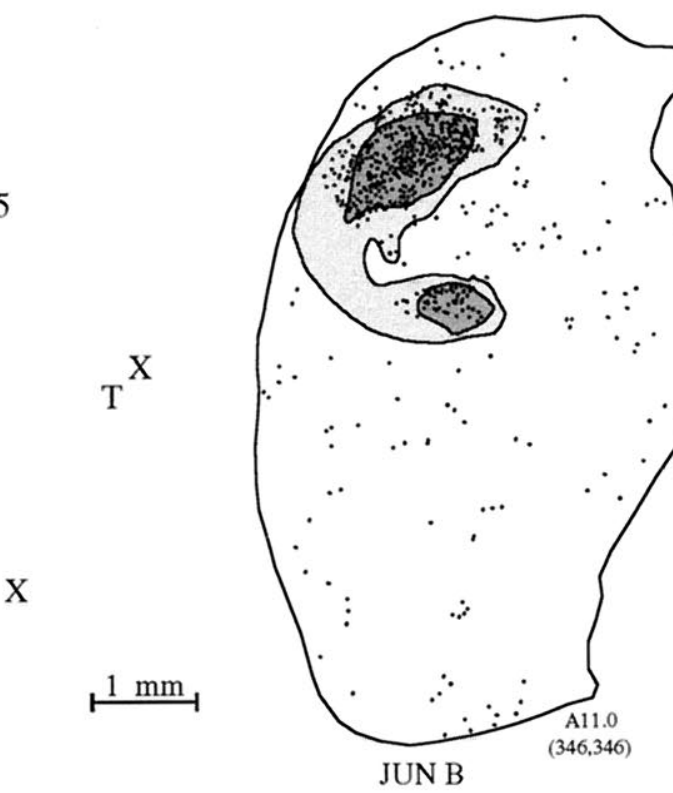

Electrical stimulation

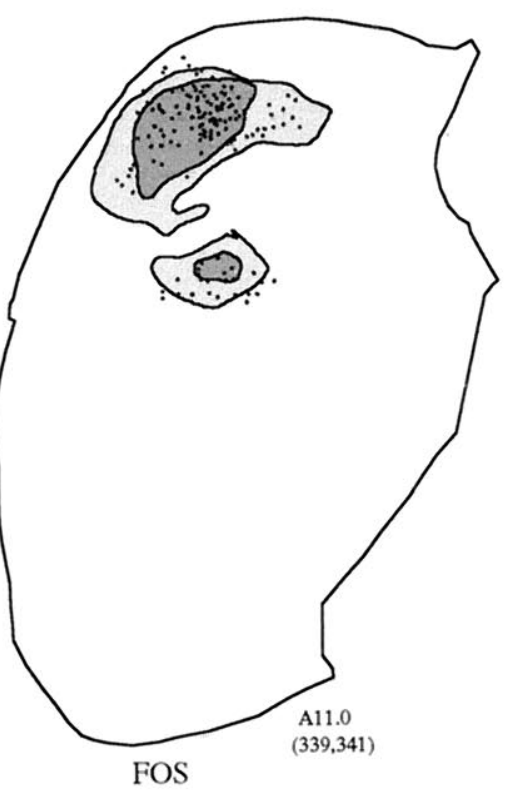

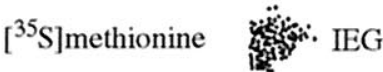

Figure 2. Electrical stimulation of the cutaneous foot digit representation in area SI induces Fos and Jun B in multiple patches in the primate striatum that correspond sharply to the afferent patches labeled by injection of $\left[{ }^{35} \mathrm{~S}\right] \mathrm{methionine}$ in the $3 \mathrm{~b}$ foot digit representation. Case SMRC48R. Left, Map of sites in cortical area SI identified by neuronal recording. $A$, Arm; $D$, digit; $F$, foot; $L L$, lower leg; Occ, occiput; $P$, foot pad; $S$, shoulder; $T$, trunk; $X$, no response. Dotted line indicates the approximate border between areas $3 \mathrm{a}$ and $3 \mathrm{~b}$. Dark circle indicates the reconstructed injection site. The lighter concentric circle indicates the less intensely labeled marginal zone of the injection site. Middle, Camera lucida chart of Jun B-positive nuclei (black dots) and anterograde $\left[{ }^{35}\right.$ S]methionine labeling (shaded regions) in the same transverse section through the putamen, processed for immunohistochemistry and autoradiography. Right, Superimposed camera lucida charts of Fos-positive nuclei and $\left[{ }^{35}\right.$ S $]$ methionine anterograde labeling in transverse sections separated by a single intervening $40 \mu \mathrm{m}$ section. Intensely labeled fiber patches (dark shading) are surrounded by zones of weaker label (light shading). Coordinates under each section indicate the approximate anteroposterior (AP) values for Saimiri sciureus (Gergen and MacLean, 1962) and the actual section numbers.

in SI, placing one electrode at the site of tracer injection in area $3 b$, and the second electrode in the foot region of area 1.

Autoradiographically labeled fibers were found in extended rostrocaudal and mediolateral arrays, which in individual transverse sections appeared as multiple patches in the dorsolateral putamen (Fig. 1A). As expected from previous work, these patches lay in the extrastriosomal matrix and thus correspond to input matrisomes (Flaherty and Graybiel, 1991, 1993a; Graybiel et al., 1993). With extended autoradiographic exposure times, these patches were seen as intensely labeled hot spots surrounded by more weakly stained halos of anterograde labeling. Fos and Jun B were strongly induced in dense clusters of neurons (density of Fos-immunoreactive nuclei $>16,000$ nuclei $/ \mathrm{mm}^{3}$ ).

There was a striking correspondence between the distributions of Fos- and Jun B-labeled nuclei and the distribution of the methionine-labeled afferents (Fig. 1). The densest accumulations of neurons expressing Fos- and Jun B-like proteins were in the hot spots of autoradiographic labeling. Some IEG-positive nuclei also were in the surrounding halo regions. Beyond these autoradiographically labeled afferent distribution zones, there were almost no Fos-labeled nuclei and only background levels of Jun B expression. Thus, the dense, patchy zones of afferent labeling matched the zones of dense IEG induction, and zones without above-background afferent labeling also lacked above-background IEG induction.

Figure 2 illustrates the results in a second experiment (SMRC48R), in which we again mapped and injected the foot representation of area $3 \mathrm{~b}$. We stimulated across the D2-5 digit representations after having injected $\left[{ }^{35} \mathrm{~S}\right]$ methionine into the D1 and dorsal foot representation, which lay farther lateral in SI. Stimulation in this case resulted in a somewhat sparser distribution of Jun B and a much sparser distribution of Fos (density of Fos $<4000$ nuclei $/ \mathrm{mm}^{3}$ ) than the distributions found in SMRC46. Nevertheless, there was a close spatial correspondence between the IEG induction and anterogradely labeled corticostriatal fiber distributions. The same sections processed for Jun B were also processed by autoradiography. These sections demonstrated a patch-for-patch overlap of the dense clusters of Jun B-positive neurons and the intense patches of labeled corticostriatal fibers (Fig. 2). The halo regions of afferent labeling had more sparse distributions of Jun B-labeled nuclei, but these levels were still above background. The sparsely distributed Fos-positive nuclei covered more of the halo regions of the corticostriatal fiber fields than did the Fos distributions found in the case illustrated in Figure 1, and some Fos-positive nuclei lay just at the edges of the halos. This slight offset may have been attributable to the fact that the sections processed for Fos and for autoradiography were separated by an intervening $40 \mu \mathrm{m}$ section.

\section{Comparison of IEG induction and afferent labeling from identical sites in SI cortex}

In the cases just described, we found a high degree of correspondence between the distribution of IEG induction and afferent fiber labeling, even though we compared the results of stimulating 


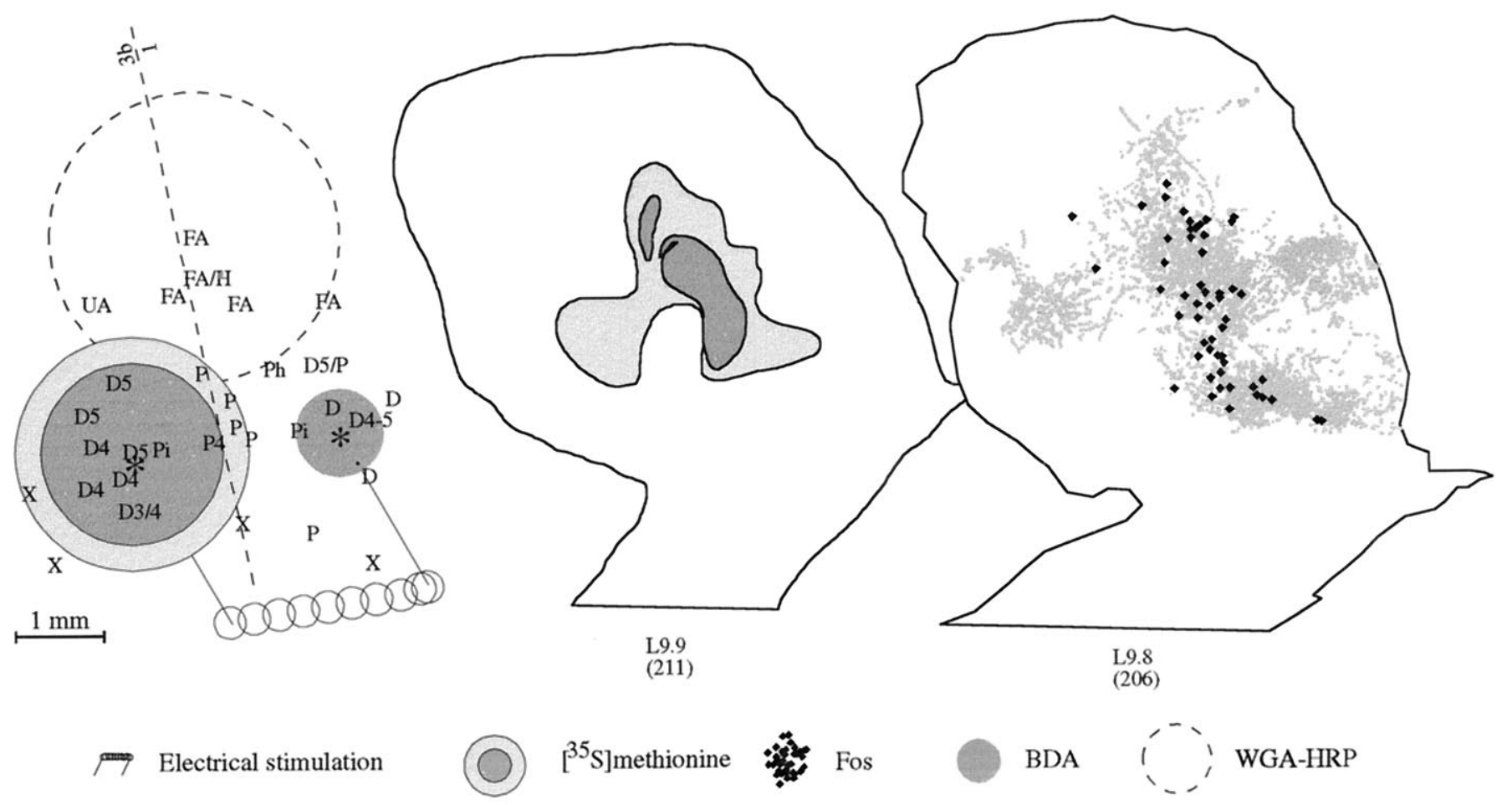

Figure 3. Parallel bipolar electrical microstimulation across the cutaneous hand digit representation in area SI induces Fos in the putamen in patchy regions corresponding to the convergence zones of corticostriatal fibers labeled from injections at each electrode. Left, Map of sites in cortical area SI identified by neuronal recording. $D$, Digit (1-5 = radial-ulnar); $F A$, forearm; $H$, hand; $P$, hand pad; $P h$, hypothenar foot pad; $P i$, insular foot pad: $U A$, upper arm; $X$, no response. Dotted line indicates the approximate border between areas $3 \mathrm{~b}$ and 1 . Concentric darkly and lightly shaded disks indicate the ${ }^{35}$ S $]$ methionine injection site (intense and marginal zones). The single dark circle indicates the reconstructed BDA injection site. The outlined circle indicates the WGA-HRP injection site. Camera lucida charts of corticostriatal fiber projections in sagittal sections through the putamen labeled by (left) injection of $\left[{ }^{35} \mathrm{~S}\right]$ methionine and (right) the distribution corticostriatal terminal varicosities labeled by injection of BDA (gray) and the distribution of Fos-positive nuclei (black dots) stained in the same section (right). There was no appreciable labeling from the WGA-HRP injection in the planes of section represented here. Coordinates under each section indicate the approximate AP values and numbers of the sections illustrated.

between two electrodes with the results of injecting a single, topographically related site. In practice, there are technical difficulties in aligning parallel bipolar stimulation and a single injection site. Exactly straddling the injection site with the stimulating electrodes would not be ideal, because the current density would be highest at the two poles.

To examine the correspondence between afferent innervation and IEG expression stimulated from identical cortical sites, we used two additional anterograde tracers, BDA and WGA-HRP. BDA has the significant advantage of having a much higher resolution than the other tracers available for use in our experiments, allowing individual fibers and varicosities to be readily identified at high magnification (see Fig. $8 A$ ). With three tracers, we were able to place each electrode at the center of an injection site and compare the IEG induction with the distribution of fibers labeled from each site. Further, we were able to compare these induction and afferent patterns with afferent distributions labeled from a neighboring, nonstimulated site injected with the third tracer.

In case SMRC49 (Fig. 3), we mapped the hand/forearm representation of area SI and placed BDA in the digit representation of area $1,\left[{ }^{35} \mathrm{~S}\right]$ methionine in the digit representation of area $3 \mathrm{~b}$, and a third tracer, WGA-HRP, into the forearm region of area $3 \mathrm{~b}$ and 1 . The stimulating electrodes were placed in the cortical sites corresponding to the $\left[{ }^{35} \mathrm{~S}\right]$ methionine and BDA injections sites.

The BDA injection in area 1 was $<1 \mathrm{~mm}$ in diameter (Fig. 3). In the ipsilateral putamen, terminal varicosities labeled with BDA were densely distributed in ventrolateral patches separated by long, sparsely branching fibers. The BDA-labeled projection field extended $>1.5 \mathrm{~mm}$ mediolaterally and $1.0 \mathrm{~mm}$ anteroposteriorly. The diameter of the $\left[{ }^{35} \mathrm{~S}\right]$ methionine injection site in area $3 \mathrm{~b}$ was twice the size of the BDA injection. The $\left[{ }^{35} \mathrm{~S}\right]$ methionine-labeled fiber projection within the striatum overlapped the BDA-labeled field but was correspondingly larger, extending farther dorsomedially. The WGA-HRP injection was large, nearly $3 \mathrm{~mm}$ in diameter, and did not overlap the BDA site and only slightly encroached on the $\left[{ }^{35} \mathrm{~S}\right]$ methionine injection site. The main WGAHRP labeling in the putamen did not overlap the BDA- or $\left[{ }^{35} \mathrm{~S}\right]$ methionine-labeled fields, but instead, appeared considerably medial to them (data not shown).

Because three different tracers were used in the experiment, striatal sections for IEG analysis were limited, and we therefore confined the analysis to immunostaining for Fos-like immunoreactivity. Stimulation across the BDA and $\left[{ }^{35} \mathrm{~S}\right] \mathrm{methionine}$ injection sites induced Fos expression in neurons within the striatal distribution fields labeled by these two tracers (Fig. 3). Indeed, although the Fos-positive neurons were sparsely distributed in the putamen $\left(<4000\right.$ nuclei $\left./ \mathrm{mm}^{3}\right)$, they were located at the centers of the clusters of BDA-labeled terminal varicosities and also matched the hot spots of autoradiographically detected $\left[{ }^{35} \mathrm{~S}\right] \mathrm{me}$ thionine labeling (Fig. 3). The most medial of the Fos-positive nuclei were along the edges of the BDA terminal field, but they still were centered within the field of $\left[{ }^{35} \mathrm{~S}\right]$ methionine-labeled 

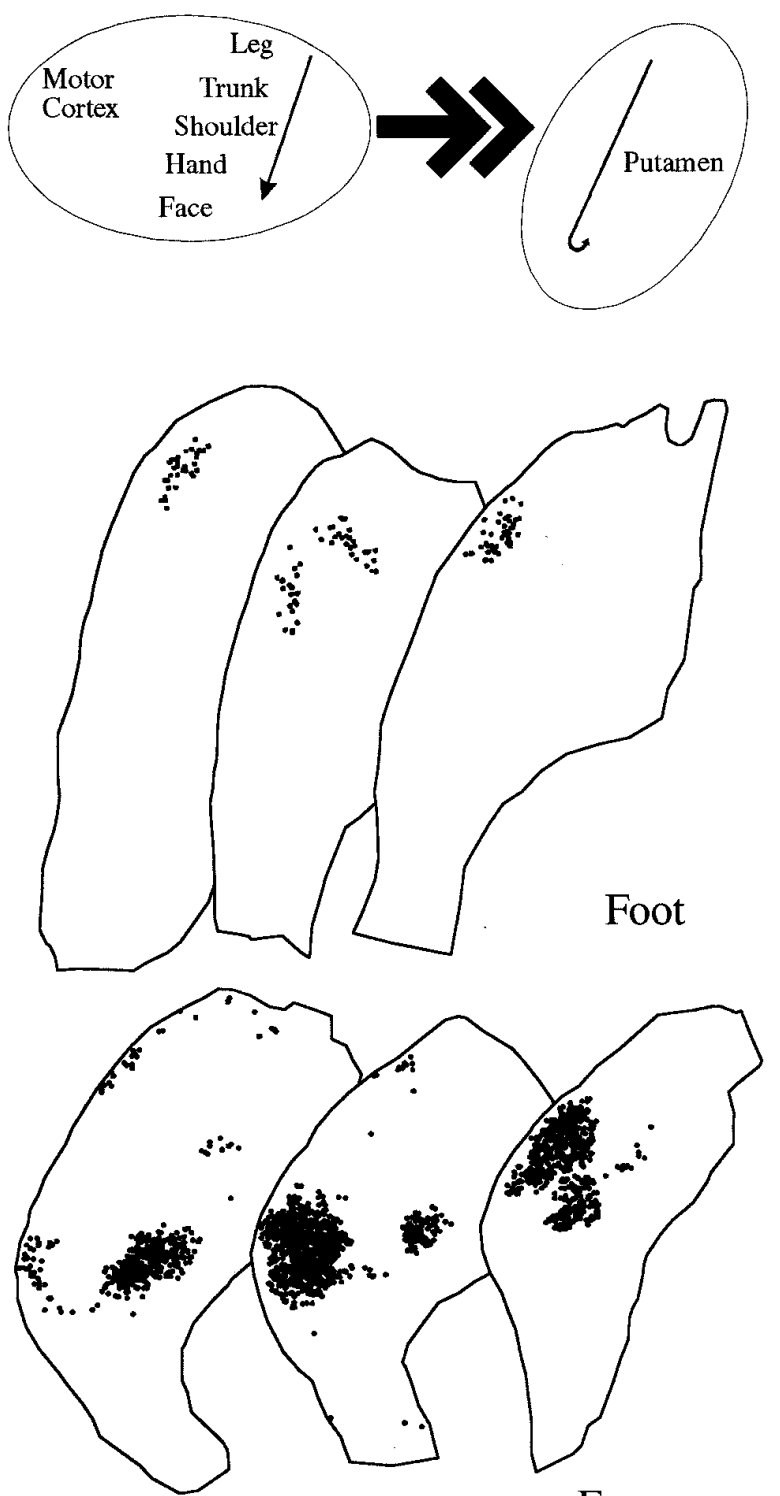

Forearm

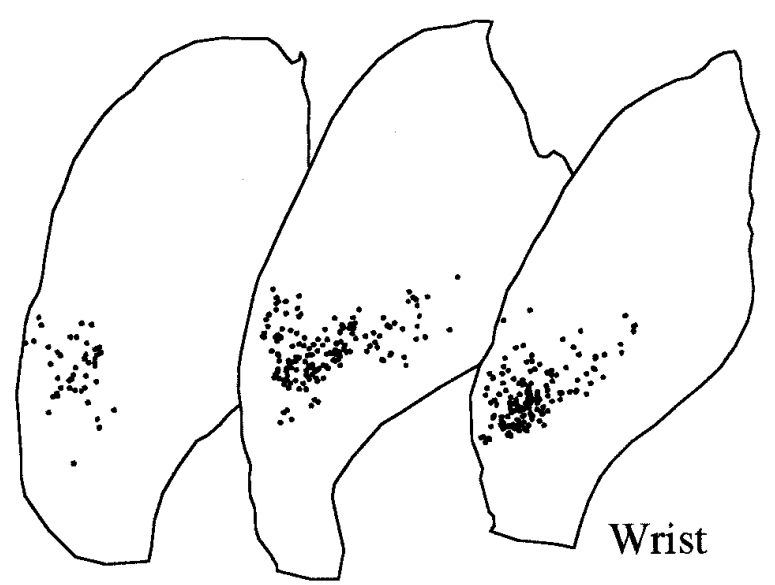

Figure 4. Electrical stimulation of sites in the MI cortex induces the expression of Fos in clusters of neurons distributed according to the known somatotopy of corticostriatal projections in the squirrel monkey (see schema at top). Cases SMRC43, SMRC44, SMRC51. Camera lucida charts of transverse sections through the putamen shown are separated by $\sim 1 \mathrm{~mm}$. In SMRC43, stimulation of a medial site in MI produced projection. There was no overlap between the region of Fos induction and the input field labeled with WGA-HRP from the nonoverlapping cortical WGA-HRP injection site.

\section{Somatotopic organization of IEG induction in the putamen stimulated from MI cortex}

In four hemispheres, we electrically stimulated regions of MI to induce IEG expression in the striatum. For each experiment, we chose an intensity of stimulation sufficient to obtain discrete movements reliably throughout the stimulation period. In this series of experiments, we made an explicit attempt to test the fidelity of somatotopic ordering of the induced IEG expression with the elicited movements as functional somatotopic markers.

Figure 4 shows charts of Fos-positive nuclei in three hemispheres in which stimulation was applied, respectively, at the foot, forearm, and wrist representations. A simple foot-up, hand-down topography is apparent, conforming to the somatotopy reflected in corticostriatal projections to the squirrel monkey putamen (Flaherty and Graybiel, 1991, 1993a). In each case, the Fospositive nuclei formed distributed arrays of more or less focal clusters in individual sections. As found for stimulation of the SI cortex, the intensity of Fos-like immunoreactivity per nucleus and the density of Fos-positive nuclei in the affected regions of the putamen varied considerably among the cases. Jun B-like immunoreactivity was expressed in patterns closely corresponding to that of Fos-like immunoreactivity, except that the Jun B labeling was denser and accompanied by background levels of Jun B expression.

In spite of the well-documented contralateral corticostriatal projection from MI cortex (Jones et al., 1977), very little induction of IEGs was elicited in the contralateral hemisphere by stimulation of MI in any of the four cases.

\section{Comparison of IEG distribution induced by stimulation of MI cortex with input matrisomes labeled from SI cortex}

In two cases, we compared the distribution of IEG expression induced by stimulation of the MI cortex with input matrisomes labeled by anterograde tracer injections in SI cortex.

In case SMRC44 (Fig. 5), we injected the hand/forearm representation of area SI with BDA and stimulated the MI cortex to induce flexions of the forearm. The Fos expression activated in the putamen by this stimulation was strong and focally dense $\left(>12,000\right.$ nuclei $\left./ \mathrm{mm}^{3}\right)$, and the distribution of Fos-positive nuclei followed closely the distribution of terminals labeled from SI (see Fig. $8 A$ ). Jun $\mathrm{B}$ distributions were similar. The only failures of match between the input fibers and zones of gene induction were in small outlying patches of densely labeled varicosities in which there was no induction of either Fos (Fig. $5 A$ ) or Jun B (data not shown).

The results in this case confirm the great fidelity in overlap of corticostriatal afferent fiber distributions labeled from SI or MI and the distributions of striatal neurons induced to express IEGs on stimulation of somatotopically corresponding SI or MI sites. To test the resolution of the somatotopic match, it was necessary

movements of the foot and induced Fos in patches in the dorsolateral putamen. In SMRC44, stimulation of a more lateral site in MI produced movements of the forearm and induced Fos in patches located more ventrally in the putamen. In SMRC51, stimulation of a yet more lateral site in MI elicited movements of the wrist and induced Fos in patches located still farther ventrally in the putamen. 

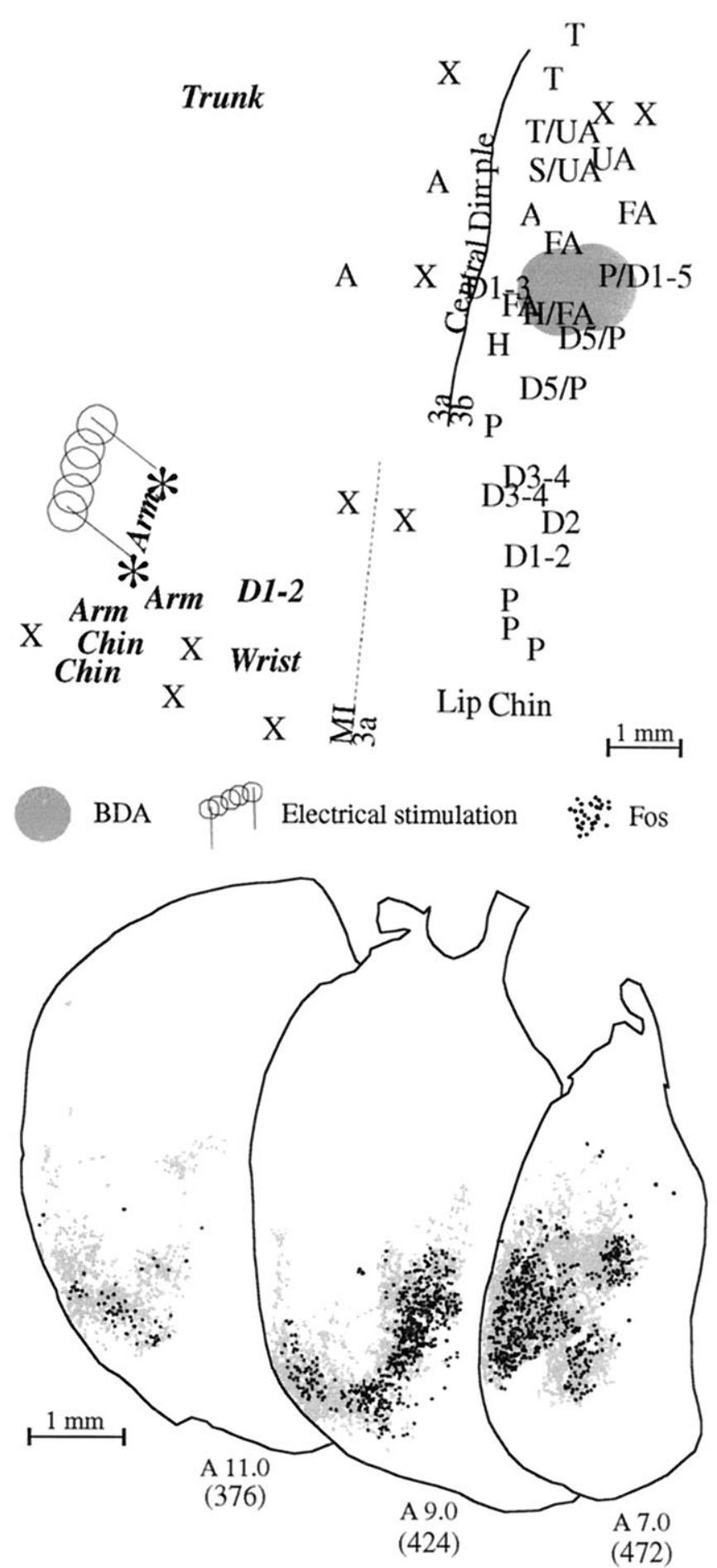

Figure 5. Electrical stimulation of the arm representation of the MI cortex induces Fos in multiple patches in the primate striatum that correspond to the distributions of terminal varicosities labeled by injection of BDA in the arm representation of the SI cortex. Case SMRC44. Top, Map of sites in cortical area SI identified by neuronal recording and in area MI identified by microstimulation. $A$, Arm; $D$, digit; $F A$, forearm; $H$, hand; $S$, shoulder; $T$, trunk; $U A$, upper arm; $X$, no response. Dotted line indicates the approximate border between areas MI and 3a. The border between $3 \mathrm{a}$ and $3 \mathrm{~b}$ is approximately along the central dimple (solid line). The dark disk indicates the reconstructed injection site. Bottom, Camera lucida charts of the distribution of corticostriatal terminal varicosities labeled by injection of BDA ( gray) and the distribution of Fos-positive nuclei (black) visualized on the same section. Coordinates indicate the AP levels and section numbers. to pair noncorresponding injection and stimulation sites. Therefore, in case SMRC43 (Fig. 6), we electrically stimulated MI cortex representing the foot and injected $\left[{ }^{35} \mathrm{~S}\right]$ methionine into SI (area $3 b$ ) cortex representing the shoulder. The distribution of Fos and Jun B in the dorsolateral putamen was sparse (density of Fos-positive nuclei $<4000$ nuclei $/ \mathrm{mm}^{3}$ ) but was typically patchy and dorsally distributed as found for stimulation of the foot representation of SI (Fig. 2). Anterograde labeling from the shoulder representation of area $3 b$ was mainly ventral to the distribution of immunopositive nuclei both for Jun B (Fig. 6) and for Fos (data not shown). In rostral sections, the anterograde tracer and IEG protein distributions were separated by $>1 \mathrm{~mm}$, and the hot spots of each were several millimeters apart. In caudal sections, the two distributions abutted and slightly overlapped for Jun, but not Fos, primarily in the halo regions of autoradiographic labeling. This differential patterning at rostral and caudal striatal levels has been noted before (Flaherty and Graybiel, 1991; Brown and Feldman, 1993).

\section{Induction of Fos and Jun B expression in other nuclei}

In one monkey (SMRC46), we processed almost every section for immunohistochemistry for Fos or Jun B. This protocol gave us the opportunity to test systematically for induction of these proteins in other structures linked to corticostriatal-basal ganglia circuitry. Stimulation of area $3 \mathrm{~b}$ cortex representing the foot produced strong expression of both transcription factors in multiple cell clusters in the putamen and, in addition, in other direct targets of the SI cortex including (1) a discrete zone in the ventroposterior lateral thalamic nucleus (VPL), (2) the dorsolateral quadrant of the subthalamic nucleus, and (3) a crescent of the reticular nucleus of the thalamus at the level of VPL labeling. As shown in Figure 7, this thalamic and subthalamic induction of the IEGs was found in regions labeled anterogradely from the corresponding cortical tracer injection.

Fos and Jun B expression was also induced in pallidal neurons distributed in a localized zone within the external segment of the globus pallidus (Fig. 7), a structure that does not receive direct afferent input from the cerebral cortex, but rather, is a major target of striatal outflow. Notably, no Fos- or Jun B-positive nuclei were observed in the internal segment of the globus pallidus or in the substantia nigra, the other major nuclei receiving direct synaptic input from the striatum.

\section{Phenotypic identification of striatal neurons expressing Fos following microstimulation of sensorimotor cortex}

To identify the neurons activated in response to cortical stimulation, we performed dual-antigen immunohistochemistry on sections from four hemispheres. We studied Fos rather than Jun B because of the almost complete lack of detectable constitutive expression of Fos-like immunoreactivity in the putamen. We were able to stain the four major types of striatal interneurons: the ChAT-containing cholinergic neurons, the parvalbumincontaining GABAergic neurons, the calretinin-containing GABAergic neurons, and the nitric oxide synthase-containing somatostatin-expressing neurons. We were only able to stain one of the two main types of striatal projection neurons (those expressing enkephalin-like immunoreactivity). Despite repeated attempts, we did not succeed in staining the dynorphin/substance $\mathrm{P}$ immunoreactive neurons. 

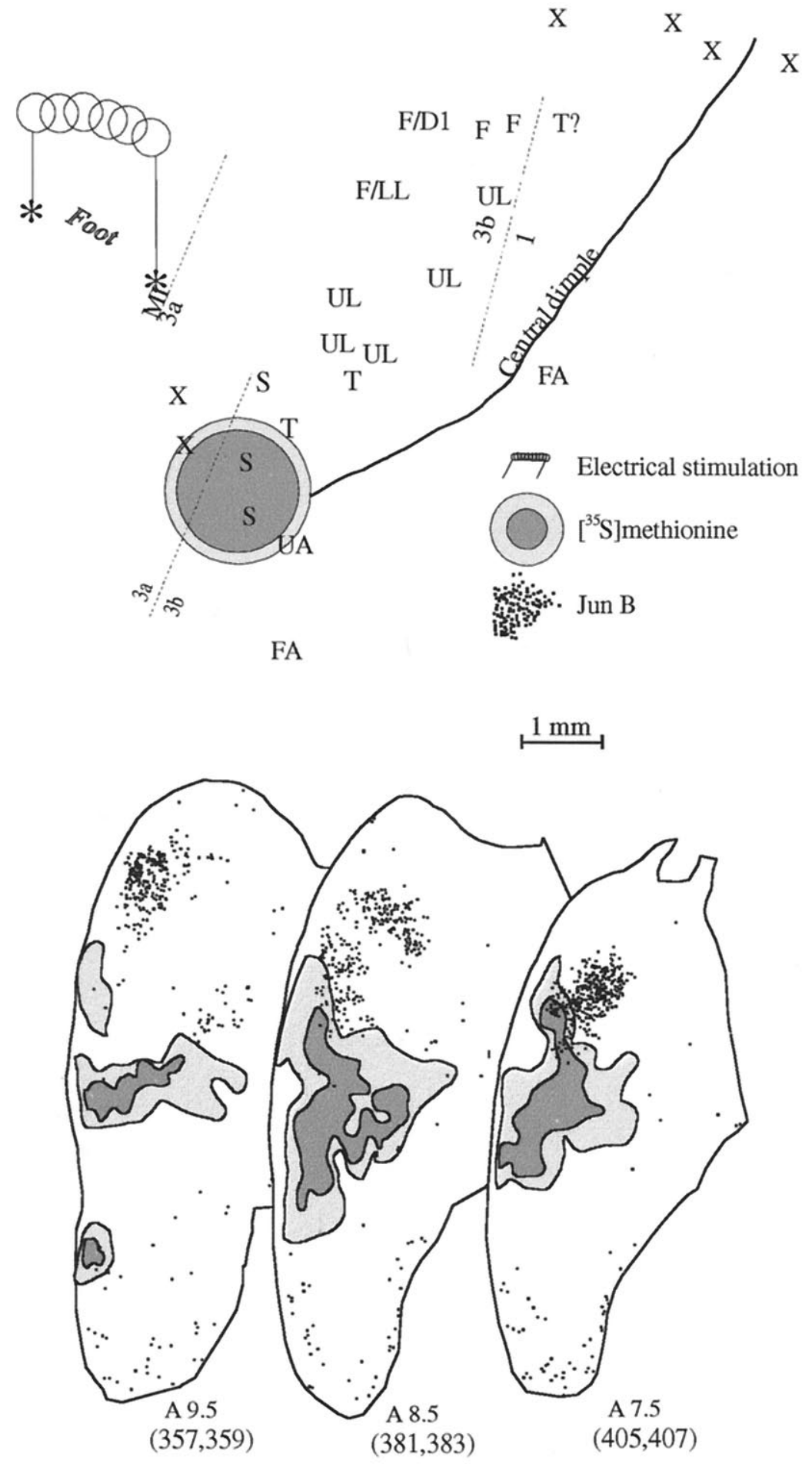

Figure 6. Electrical stimulation of the foot representation of the MI cortex induces Jun B in multiple patches in the primate striatum that do not overlap the afferent patches labeled by injection of $\left[{ }^{35} \mathrm{~S}\right]$ methionine in the shoulder representation in area 3b. Case SMRC43. Top, Map of sites in cortical area SI identified by neuronal recording. $D$, Digit; $F$, foot; $F A$, forearm; $L L$, lower leg; $S$, shoulder; $T$, trunk; $U A$, upper arm; $U L$, upper leg; $X$, no response. Dotted lines indicate the approximate borders between areas MI and 3a, $3 \mathrm{a}$ and $3 \mathrm{~b}$, and $3 \mathrm{~b}$ and 1 . Darkly and lightly shaded disks indicate, respectively, the strongly labeled and the less intensely labeled marginal zones of the injection site. Bottom, Superimposed camera lucida charts of Jun B-positive nuclei (black dots) and anterogradely labeled fibers (shading) from transverse sections separated by an intervening $40 \mu \mathrm{m}$ section. Intensely labeled fiber patches (dark shading) are surrounded by zones of weaker label (pale shading). Coordinates under each section indicate the approximate AP levels and numbers of the sections.
Fos induction in parvalbumin-containing

GABAergic interneuron

Exquisite sensitivity of stimulation-induced Fos expression was found in the parvalbumin-containing interneurons of the striatum. In hemispheres in which cortical stimulation resulted in only very sparse IEG induction in the putamen, almost all Fos-containing nuclei were found in these interneurons. This extreme responsivity of the parvalbumin-containing neurons was dramatically demonstrated in case SMRC46R, in which stimulation in the hand representation of MI induced expression of Fos immunoreactivity in the ventrolateral putamen. Approximately five nuclei per section were Fos-immunoreactive, and all were in parvalbuminpositive neurons (Fig. 8A). In case SMRC51 (Fig. 4), in which stimulation of the hand area of MI induced stronger expression of Fos-like immunoreactivity (density $<2000 / \mathrm{mm}^{3}$ ), fully $84 \%$ of the strongly labeled nuclei were found in parvalbumin-containing neurons (146/174). Only when the much more weakly Fosimmunoreactive nuclei were counted, which would probably have been masked by double-labeling of the perikarya, did the percentage of co-localization for the parvalbumin interneurons drop, and 


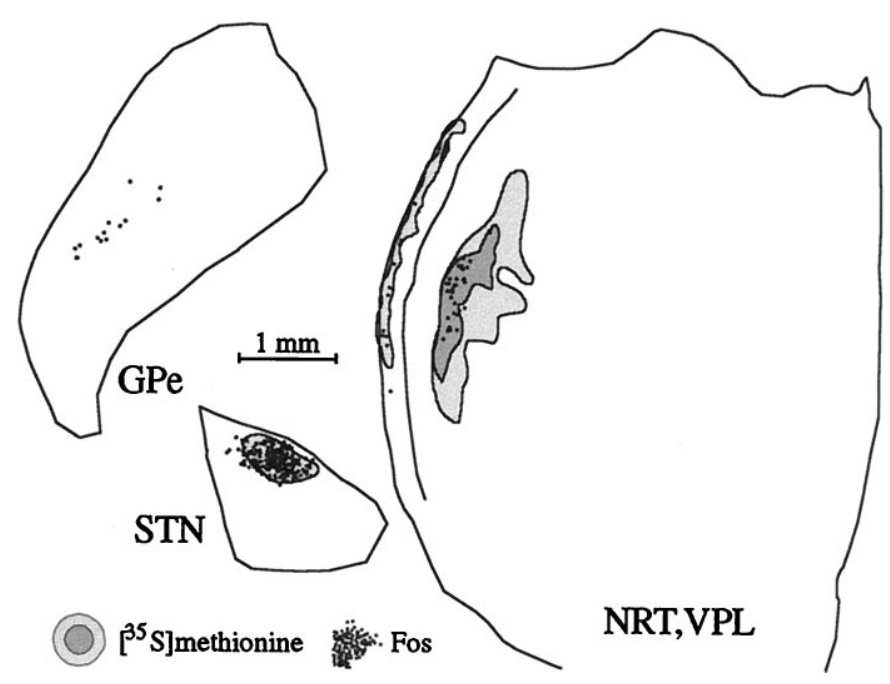

Figure 7. Electrical stimulation of the foot representation of SI area $3 b$ induces the expression of Fos in neurons of the thalamus, subthalamic nucleus, and external segment of the globus pallidus (case SMRC46L). Black dots illustrate the distribution of Fos-positive nuclei. Shaded regions indicate labeling traced from injection of $\left.{ }^{35} \mathrm{~S}\right]$ methionine at the site of stimulation. NRT, Reticular nucleus of the thalamus; $G P e$, external segment of the globus pallidus; $S T N$, subthalamic nucleus; VPL, ventral posterolateral nucleus of the thalamus.

even then, the parvalbumin interneurons, which themselves are very sparsely distributed relative to striatal projection neurons, accounted for $>50 \%$ of the Fos immunolabeling (53\%).

Neither the sparse expression of Fos-like immunoreactivity nor the predominance of Fos expression in parvalbumin-containing interneurons was limited to stimulation of MI cortex (nor to stimulation of cortex representing the hand). For example, in case SMRC48R (Fig. 9A), stimulation in the foot representation of area $3 \mathrm{~b}$ induced relatively weak Fos expression (density $<4000$ nuclei $/ \mathrm{mm}^{3}$ ), and yet $75 \%$ of these (180/241 counted) were in parvalbumin-containing neurons.

The distributions of the Fos-positive parvalbumin interneurons followed the patterns already described for other cases. They followed a foot-up, hand-down pattern in the putamen and were in partly interconnected clusters. It was striking that the nonparvalbumin containing Fos-positive neurons in these cases were intermingled with the parvalbumin-positive neurons and often had distributions that extended beyond those of the parvalbuminpositive neurons. In many sections, this gave the impression that the parvalbumin-negative Fos-labeled cells formed small clusters within the larger clusters of Fos-labeled parvalbumin-positive neurons (Fig. 9).

There was an equally striking distribution of Fos-labeled parvalbumin interneurons when the induction of Fos expression in the putamen was intense. This is shown in Figure 9, $B$ and $C$, for case SMRC46L, in which electrical stimulation applied to the foot representation of area $3 \mathrm{~b}$ induced very strong expression of Fos in the putamen (density $>16,000$ nuclei $/ \mathrm{mm}^{3}$ ). Although they came to be a minority of all neurons expressing Fos-like immunoreactivity ( $\ll 20 \%$ ), virtually every parvalbumin-containing neuron in the field of induction strongly expressed Fos $(95 \%, 275 / 291$; Fig. $8 C$ ). Fos-positive parvalbumin-negative neurons again tended to lie within the distribution zones of the parvalbumin-containing Fos-positive neurons and tended to form clusters inside them. Thus, the local intracortical stimulation typically induced a pattern of Fos expression in which Fos-positive parvalbumin inter- neurons formed patchy fields within which there were, in addition, dense foci of parvalbumin-negative neurons (see example at high magnification in Fig. 9C).

\section{Fos induction in other striatal interneurons}

In cases with very weak induction of Fos-like immunoreactivity, the parvalbumin-containing interneurons were the only, or nearly the only, interneuronal cell type activated to express Fos in response to stimulation of sensorimotor cortex. For example, in case SMRC51 described above, the parvalbumin-immunoreactive interneurons were the only Fos-positive interneurons we observed. No Fos co-localization was present in neurons stained for NADPH diaphorase, calretinin, or ChAT. However, in SMRC46L, in which induction of Fos was strong and dense, $60 \%$ (30/50) of NADPH diaphorase-containing neurons in the field of induction expressed Fos-like immunoreactivity (Fig. 8D). NADPH diaphorase-containing neurons in the center of the fields of induction almost always expressed Fos, whereas the likelihood of co-localization dropped at the peripheries of the fields. Thus, the composite percentage value of $60 \%$ combined a near $100 \%$ value at the center of the Fos-positive clusters and a much lower value near the edges of the clusters. Only $12 \%(18 / 153)$ of the calretinin-containing neurons were found to express Fos (Fig. $8 E$ ), and, in contrast to the NADPH diaphorase-containing neurons, these were not concentrated at the centers of the fields of induction, but scattered through them. Fos-like immunoreactivity was not expressed in any of the cholinergic interneurons we analyzed $(n=103$, Fig. $8 F)$.

\section{Induction of Fos-like immunoreactivity in enkephalin-immunoreactive neurons}

To analyze IEG induction in striatal projection neurons, we performed double immunostaining for Fos-like immunostaining and enkephalin-like immunoreactivity in three hemispheres, SMRC46L and SMRC44, and SMRC51. In case SMRC51, as expected from the analysis of Fos expression in striatal interneurons, only occasional enkephalin-positive cells were found to express Fos. However, in SMRC46L and SMRC44, in which Fos expression was intense (Fig. 8G) and interneurons accounted for only a small fraction of the Fos expression, Fos and enkephalin were highly co-localized. We calculated the percentages of all Fos-positive nuclei that were in enkephalin-immunoreactive neurons to obtain a value for the responsivity of these projection neurons, which constitute approximately half the projection neurons in the striatum. Because we had no successful immunomarker for the other (dynorphin/substance $\mathrm{P}$ immunoreactive) projection neuron class, we made estimates of the probable amount of labeling of these neurons by taking into account the values for the enkephalin-positive neurons and the density and labeling values for the four interneuronal types discussed above. Both in case SMRC46L, in which the cortical stimulation was of the SI area $3 b$ foot representation, and in case SMRC44, in which MI forearm representation was stimulated, $\sim 75 \%$ of all Fos-positive nuclei were in enkephalin-immunoreactive neurons (267/355 in SMRC46 and 115/150 in SMRC44). If only 5\% of the remaining Fos-positive nuclei in the regions of densest expression were found in interneurons (parvalbumin-containing and NADPHdiaphorase containing), this would leave $\sim 20 \%$ as the maximum possible labeling that could be accounted for by the dynorphin/ substance P-containing projection neurons. 

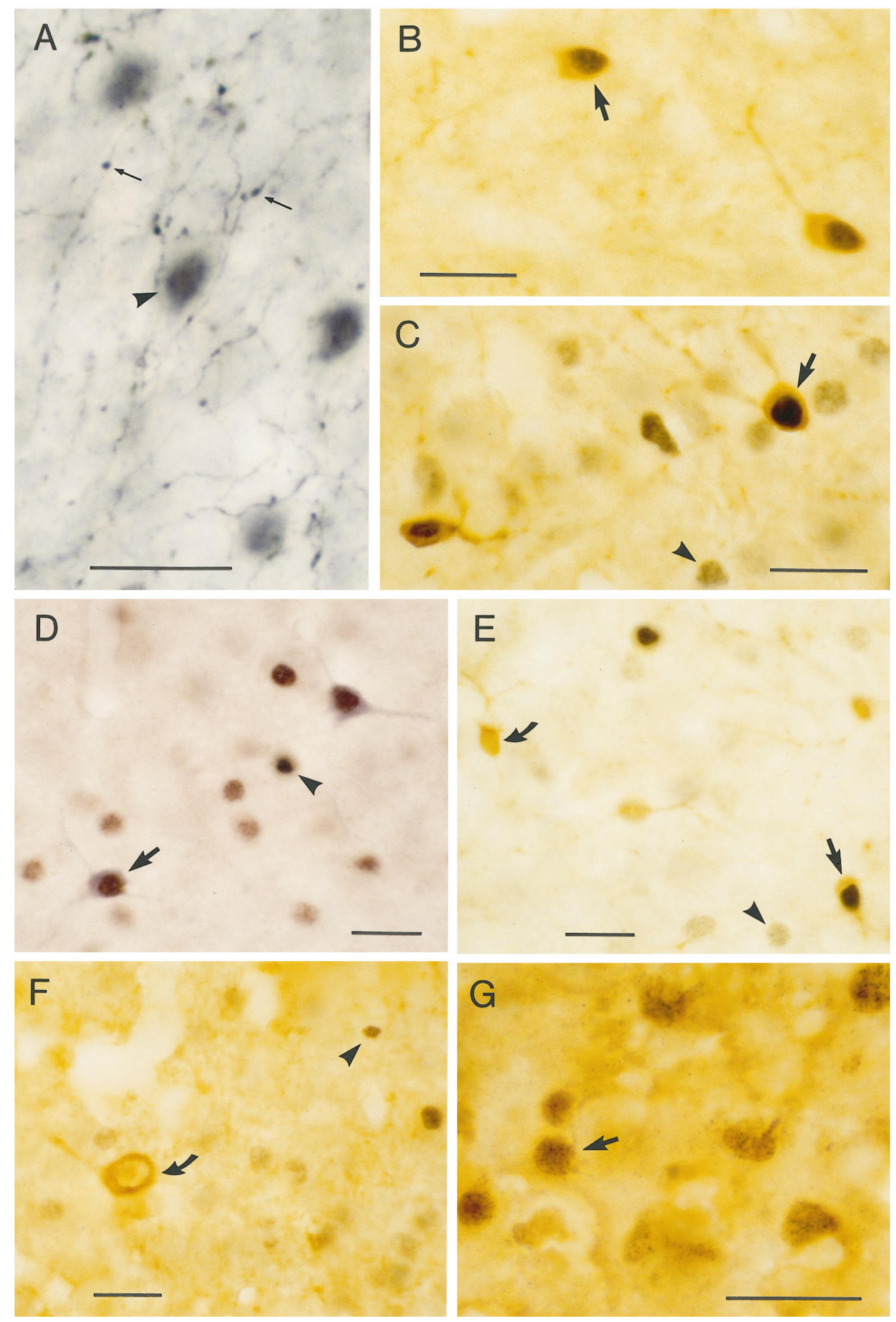

Figure 8. A, Photomicrograph of corticostriatal fibers labeled by injection of BDA and Fos-positive nuclei labeled by cortical microstimulation in case SMRC44 (see Fig. 5) taken from section 472 charted in Figure 5. Arrowhead points to a Fos-positive nucleus. Thin arrows point to varicosities in BDA-labeled corticostriatal fibers. Photomicrographs in $B$ and $C$ show that electrical stimulation of the sensorimotor cortex induces Fos expression in the parvalbumin-containing interneurons of the squirrel monkey putamen. Perikarya of parvalbumin-containing neurons are brown; Fos-positive nuclei are black. B illustrates co-localization of Fos and parvalbumin in case SMRC46R, in which Fos induction in the putamen was only found in a few cells, and $C$ illustrates co-localization in case SMRC46L, in which the Fos induction was very intense. Arrows point to examples of parvalbumin-containing neurons expressing Fos, and arrowheads point to Fos-positive nuclei not double-labeled for parvalbumin. $D-G$, Electrical stimulation of the SI cortex induces the expression of Fos in NADPH-diaphorase containing interneurons $(D)$ and occasionally in calretinin-containing interneurons $(E)$, but never in cholinergic interneurons $(F)$. The majority of Fos-positive nuclei were found to be induced in enkephalinergic neurons $(G)$. Arrows indicate co-localization of Fos-positive nuclei in the phenotypically defined striatal neurons, arrowheads indicate Fos-positive nuclei without the respective marker co-localization, and curved arrows indicate striatal neurons in which Fos was not appreciably induced. Scale bars, $20 \mu \mathrm{m}$. 

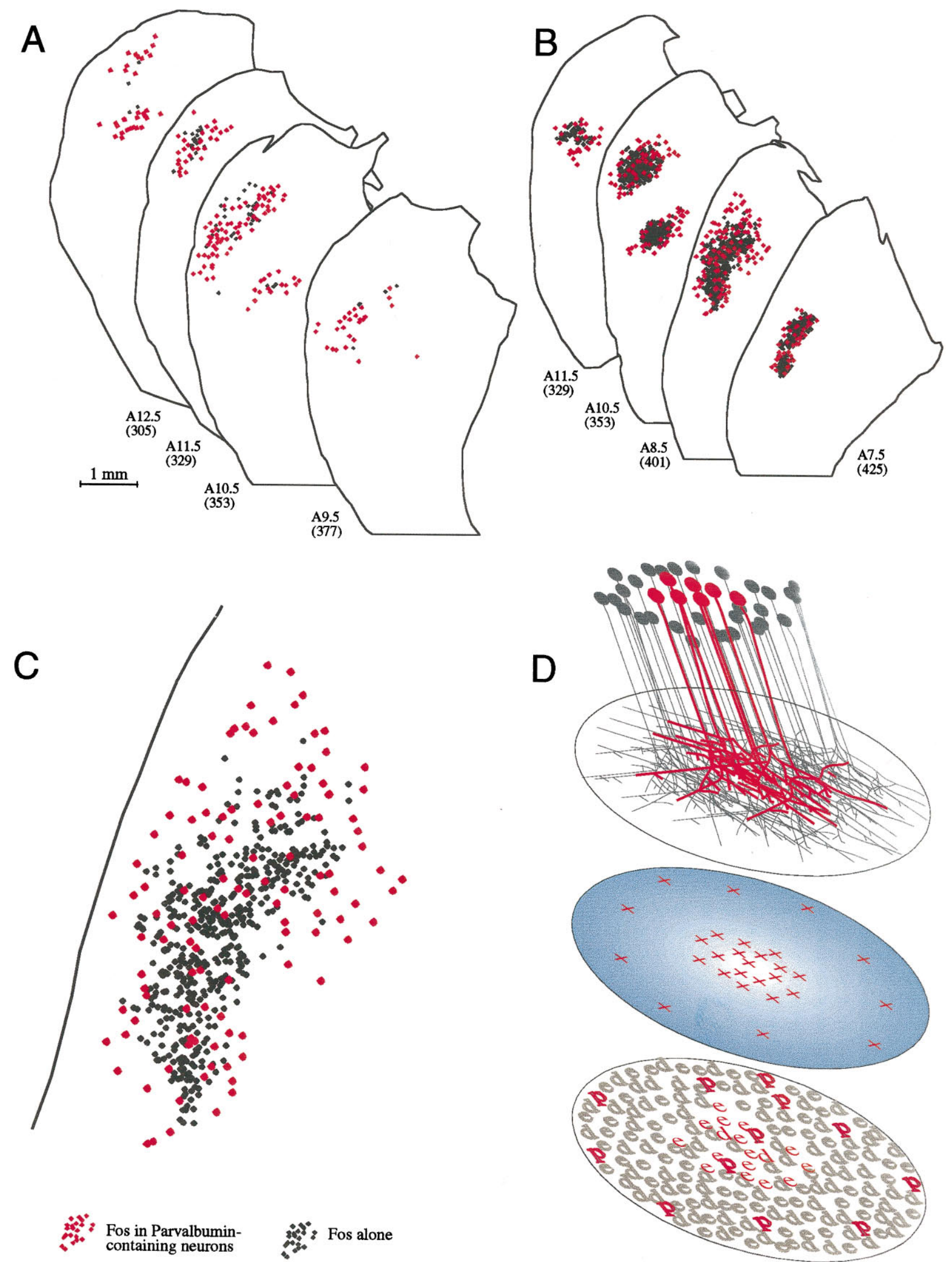

Figure 9. Distributions of parvalbumin-containing neurons expressing Fos in response to electrical stimulation of the sensorimotor cortex. $A$, Charts illustrate case SMRC48R, in which stimulation of the foot representation of SI cortex induced Fos in a relatively sparse pattern. Red dots indicate Fos co-localized with parvalbumin in striatal neurons. Black dots indicate Fos labeling not co-localized with parvalbumin. The majority of the neurons expressing Fos were found to contain parvalbumin. B, C, Case SMRC46L, in which stimulation of the foot representation of (Figure legend continues) 


\section{DISCUSSION}

Striatal neurons in alert behaving monkeys have highly contextdependent properties (Hikosaka et al., 1989; Alexander and Crutcher, 1990; Kimura, 1990; Schultz and Romo, 1992; Jaeger et al., 1993). These properties are thought to contribute to the motor control functions of the basal ganglia and to derive from selective activation of convergent inputs onto modular arrays of striatal neurons. Coherent activity of these convergent inputs is thought necessary to activate striatal projection neurons. Despite suggestive anatomical evidence for such convergent modular organization, however, no functional evidence for these models has yet been available. We used the strategy of monitoring IEG activation by cortical microstimulation as a way to examine the population response of striatal neurons to coherent activation of cortical inputs. Our findings suggest that local sites in SI and MI cortex can activate clusters of striatal neurons and raise further the possibility that the degree of convergent activation is regulated by striatal interneurons. The main targets of this cortical input, as measured in the gene induction assay, were neurons of the indirect striatopallidal pathway and subsets of striatal interneurons. This suggests that the sensorimotor cortex may selectively and dynamically regulate the release functions of the basal ganglia motor circuit.

\section{The use of IEG induction to study neuronal ensembles}

We used the gene induction assay (Sagar et al., 1988; Hughes and Dragunow, 1995) as a form of functional imaging. Like deoxyglucose-related and cerebral blood flow-related methods (Sokoloff et al., 1977; Raichle, 1994), this method lacks temporal information about neural responses and leaves open the question of how the activation measured relates to electrophysiological activity. However, such techniques share the advantage of permitting measurement of large-scale activity patterns, and the gene induction assay, in particular, offers the possibility of transneuronal cellular identification.

By stimulating both SI and MI, we tried to avoid some obvious indirect routes for the activation of striatal neurons other than the direct corticostriatal pathway. For example MI, but not SI, projects strongly to the centre median, which in turn projects to the putamen (Yeterian and Van Hoesen, 1978; Jones et al., 1979). The activation patterns we found are consistent with both the somatotopic and modular patterns of corticostriatal afferents labeled in the same experiments. We cannot, however, exclude the possibility that other routes were responsible or acted cooperatively in producing the observed striatal gene activation. In particular, antidromic excitation of corticocortical axons could have produced coherent activity in multiple cortically interconnected regions, the convergence of whose efferents might be necessary to activate striatal neurons. Indeed, the absence of induction in the contralateral putamen by MI stimulation is consistent with this idea as contralaterally interconnected homotopic MI sites mainly send nonoverlapping projections to the striatum (Flaherty and Graybiel, 1993) and should, therefore, not synergistically activate striatal neurons.

Finally, the barbiturate anesthesia used in these experiments may have been responsible for limiting the number and cell type in which Fos expression was induced. For example, deep anesthesia could have reduced cortical excitability and thereby accounted for differences in induction observed among cases for which similar regions were stimulated. However, the striking selectivity we found for corticostriatal activation of indirect pathway neurons has also been found by our group in awake freely moving rats (S. Berretta, H. Parthasarathy, and A. Graybiel, unpublished observations).

\section{Input-output matrisomes in the primate putamen}

Our results provide strong support for the notion of matrisomes as pre- and postsynaptic organizing features of the striatal matrix. BDA labeling resolved afferent fiber patches, because clusters of terminal varicosities connected with one another and to the white matter by long, thick BDA-labeled axons. These connecting fibers had few BDA-labeled varicosities. The borders of the clusters of varicosities and their matching cell clusters were thus quite sharp. Furthermore, afferent fiber clusters were closely matched by clusters of immunoreactive striatal neurons showing gene expression induced from cortical sites matching those injected with tracer, and neurons outside the afferent fiber distributions were only rarely immunoreactive for Fos or Jun B beyond constitutive levels. Thus, clusters of input fibers labeled from focal sites in sensorimotor cortex can functionally activate corresponding clusters of striatal neurons in the primate putamen.

\section{Differential activation of basal ganglia indirect pathway neurons by stimulation of sensorimotor cortex}

Our phenotypic analysis of the striatal neurons activated by stimulation of sensorimotor cortex demonstrated that most of the Fos-positive neurons in the densely labeled cell clusters were enkephalinergic projection neurons. These neurons make up approximately half of all striatal projection neurons and are the neurons that project to the external segment of the globus pallidus (Penny et al., 1986; Shivers et al., 1986; Flaherty and Graybiel, 1993b). Our quantitative estimates indicate that only a small

\section{$\leftarrow$}

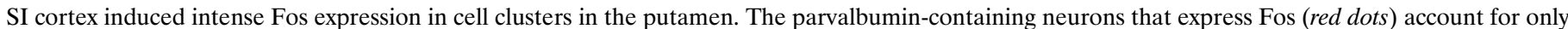

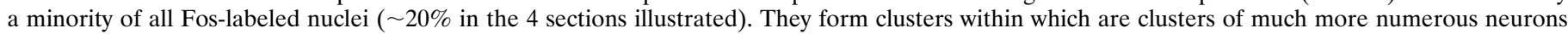

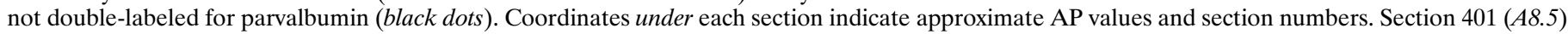

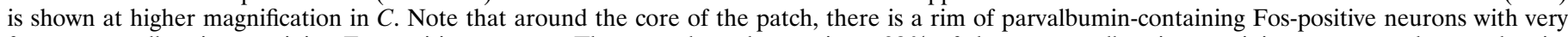

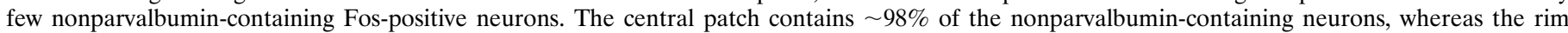

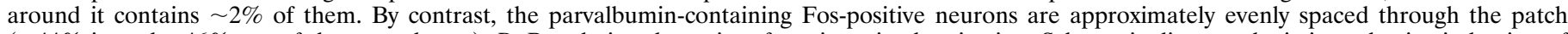

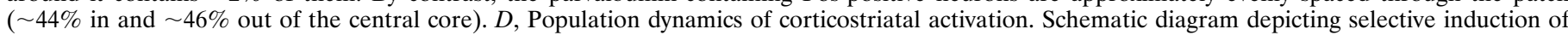

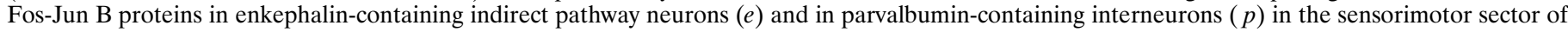

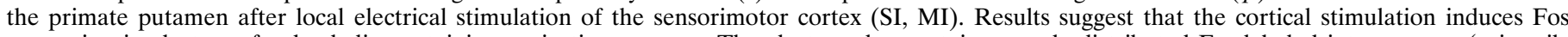

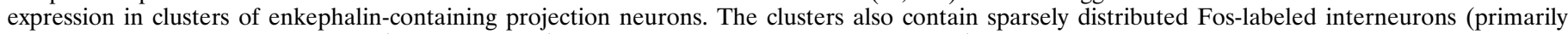

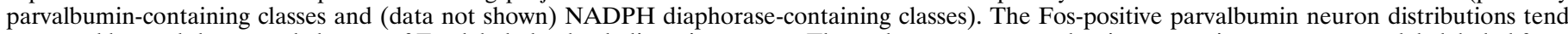

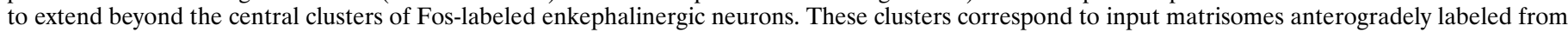

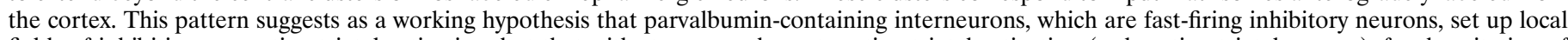

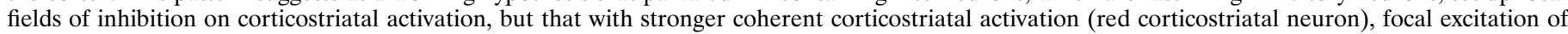

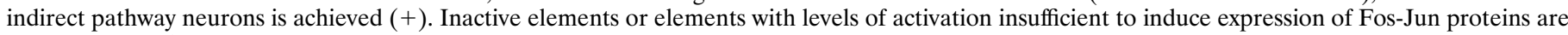
shown in gray. $d$, Dynorphin-containing direct pathway neurons. 
proportion of striatal neurons containing substance $\mathrm{P}$ and dynorphin could have expressed Fos. In a direct test of this possibility in the rat, in which we could immunostain dynorphin-containing neurons, we have found an overwhelming bias toward activation of enkephalinergic projection neurons by stimulation of sensorimotor cortex (S. Berretta, H. Parthasarathy, and A. Graybiel, unpublished observations).

Although the gene induction effects we monitored may not mirror spike activations in these neurons (see Deisseroth et al., 1996; Liu and Graybiel, 1996), they do reflect the activation of second messenger pathways that could operate at extended time scales and have effects related to neuroplasticity. Interestingly, cortical activity seems necessary for maintaining levels of enkephalin itself in indirect pathway neurons (Uhl et al., 1988), an effect that may be influenced by transcription factors of which the AP-1 binding proteins we studied here are representative (Sonnenberg et al., 1989; Konradi et al., 1993).

Consistent with specialized activation of the indirect pathway by the sensorimotor cortex, we also found transneuronal induction of Fos and Jun B in neurons of the external pallidum, but not of the internal pallidum or the substantia nigra. This pattern is similar to that observed by Strick and colleagues after injection of anterograde transneuronally transported virus into the MI cortex (Strick et al., 1995). If the pallidal IEG induction is derived from activation of striatopallidal inputs, the causal agent may be GABA or some other factor released by striatopallidal terminals such as enkephalin acting at opiate receptors (Chang et al., 1988; Maneuf et al., 1996) or a growth factor (Korsching, 1993; Peng et al., 1993). Alternatively, the IEG expression could be driven by the subthalamic nucleus, which receives inputs from MI and SI cortex, sends an excitatory projection to the globus pallidus (Parent and Hazrati, 1995), and showed IEG induction in our experiments .

Our findings demonstrate that sensorimotor cortex has selective impact on the class of striatal projection neurons that give rise to the indirect pathway of the basal ganglia. This pathway augments basal ganglia inhibition of motor centers and is the target of therapeutic attempts to increase motor function in Parkinson's disease (see Albin et al., 1989; Alexander and Crutcher, 1990; Graybiel, 1996). The cortically driven induction of Fos in enkephalinergic striatal neurons, as compared with the directly projecting striatal neurons, may be the result of differences in neurotransmitter receptors, in intracellular messenger cascade machinery, or even in density of cortical input. A possibility clearly raised by our findings is that the opponent functions of the direct and indirect pathways can be manipulated differentially by the sensorimotor cortex.

\section{A dynamic focusing mechanism for corticostriatal transmission}

Equally striking was the sensitivity of Fos induction in parvalbumincontaining interneurons to cortical stimulation. When stimulation was only weakly effective, almost all of the Fos-positive nuclei in the putamen were found in these interneurons. When the stimulation strongly induced Fos in dense clusters of neurons, parvalbumincontaining neurons appeared not only in the clusters, but also in border zones around their densely labeled centers. Thus, at the centers of the clusters, most neurons were enkephalinergic projection neurons, whereas at their margins, most activated neurons were parvalbumin-containing interneurons.

The parvalbumin-containing interneurons of the striatum are fast-spiking GABAergic neurons with very short duration action potentials, and in the rat striatum, they have been shown to be highly sensitive to depolarizing input (Kawaguchi, 1993). The apparent sensitivity of the parvalbumin neurons we saw with the gene induction assay could reflect a rapid rise in intracellular calcium with the depolarization and their fast firing. Interestingly, the parvalbumin neurons are thought to be connected through gap junctions, so that they can inhibit simultaneously a large number of their target neurons (Kita et al., 1990; Kawaguchi et al., 1995).

Given these properties, our results suggest a potential intrastriatal mechanism for adjusting the efficacy of corticostriatal transmission. According to this working hypothesis (Fig. 9D), at low levels of corticostriatal stimulation, the differentially sensitive parvalbumin interneurons could become activated and set up a distributed zone of feed-forward inhibition in the sensorimotor putamen. Only stronger coherent cortical activity would be able to break through the inhibition and activate clusters of projection neurons within the parvalbumin neurons' inhibitory field. Such an inhibitory network might be important in preventing the direct pathway from being triggered into activity by small fluctuations in cortical activity. Moreover, the activity of such a network in the sensorimotor putamen could serve as a focusing mechanism to provide relatively fast, modular activation of selected sets of striatal projection neurons. An "input matrisome" then could be defined dynamically, either by filtering cortical inputs to activate subsets of neurons within a matrisome, or alternatively, by suppressing activity in surrounding matrisomes to shape the field of neurons responsive to a given cortical signal. Either mechanism might serve in lieu of the local collateral inhibitory mechanisms, which have long been proposed but for which evidence is lacking (Jaeger et al., 1994). Such dynamic tuning could be part of the basis for context-dependent activation of striatal neurons and could indicate how selective basal ganglia release of function might be achieved despite massive corticostriatal convergence.

\section{REFERENCES}

Albin RL, Young AB, Penney JB (1989) The functional anatomy of basal ganglia disorders. Trends Neurosci 12:366-375.

Alexander GE, Crutcher MD (1990) Functional architecture of basal ganglia circuits: neural substrates of parallel processing. Trends Neurosci 13:266-272.

Besson MJ, Rogard M, Zalc B, Deniau JM (1993) Effects of cortical electrical stimulation on c-Fos expression in the basal ganglia. Soc Neurosci Abstr 19:133.

Brandt HM, Apkarian AV (1992) Biotin-dextran: a sensitive anterograde tracer for neuroanatomic studies in rat and monkey. J Neurosci Methods 45:35-40.

Brown LL, Feldman SM (1993) The organization of somatosensory activity in dorsolateral striatum of the rat. In: Progress in brain research, Vol 99 (Arbuthnott GW, Emson PC, eds), pp 237-250. Amsterdam: Elsevier.

Chang SL, Squinto SP, Harlan RE (1988) Morphine activation of c-fos expression in rat brain. Biochem Biophys Res Commun 157:698-704.

Cowan RL, Wilson CJ (1994) Spontaneous firing patterns and axonal projections of single corticostriatal neurons in the rat medial agranular cortex. J Neurophysiol 71:17-32.

Deisseroth K, Bito H, Tsien RW (1996) Signaling from synapse to nucleus: postsynaptic CREB phosphorylation during multiple forms of hippocampal synaptic plasticity. Neuron 16:89-101.

Flaherty AW, Graybiel AM (1991) Corticostriatal transformations in the primate somatosensory system. Projections from physiologically mapped body-part representations. J Neurophysiol 66:1249-1263.

Flaherty AW, Graybiel AM (1993a) Two input systems for body representations in the primate striatal matrix; experimental evidence in the squirrel monkey. J Neurosci 13:1120-1137.

Flaherty AW, Graybiel AM (1993b) Output architecture of the primate putamen. J Neurosci 13:3222-3237.

Flaherty AW, Graybiel AM (1995) Motor and somatosensory cortico- 
striatal projection magnifications in the squirrel monkey. J Neurophysiol 74:2638-2648.

Fu L, Beckstead RM (1992) Cortical stimulation induces fos expression in striatal neurons. Neuroscience 46:329-334.

Gergen JA, MacLean PD (1962) A stereotaxic atlas of the squirrel monkey's brain (Saimiri sciureus). Bethesda, MD: National Institutes of Health.

Graybiel AM (1996) Basal ganglia: new therapeutic approaches to Parkinson's disease. Curr Biol 6:368-371.

Graybiel AM, Kimura M (1995) Adaptive neural networks in the basal ganglia. In: Models of information processing in the basal ganglia (Houk JC, Davis JL, Beiser DG, eds), pp 103-116. Cambridge, MA: MIT.

Graybiel AM, Flaherty AW, Giménez-Amaya J-M (1993) Striosomes and matrisomes. In: The basal ganglia (Bernardi G, Carpenter M, Di Chiara G, Morelli M, Stanzione P, eds), pp 3-12. New York: Plenum.

Graybiel AM, Aosaki T, Flaherty AM, Kimura M (1994) The basal ganglia and adaptive motor control. Science 265:1826-1831.

Hikosaka O, Sakamoto M, Usui S (1989) Functional properties of monkey caudate neurons. III. Activities related to expectation of target and reward. J Neurophysiol 61:814-832.

Houk JC, Wise SP (1995) Distributed modular architectures linking basal ganglia, cerebellum, and cerebral cortex: their role in planning and controlling action. Cereb Cortex 5:95-110.

Hughes P, Dragunow M (1995) Induction of immediate early genes and the control of neurotransmitter-regulated gene expression within the nervous system. Pharmacol Rev 47:133-178.

Jaeger D, Gilman S, Aldridge JW (1993) Primate basal ganglia activity in a precued reaching task: preparation for movement. Exp Brain Res 95:51-64.

Jaeger D, Kita H, Wilson CJ (1994) Surround inhibition among projection neurons is weak or nonexistent in the rat neostriatum. J Neurophysiol 72:2555-2558.

Jones EG, Coulter JD, Burton H, Porter R (1977) Cells of origin and terminal distribution of corticostriatal fibers arising in the sensorimotor cortex of monkeys. J Comp Neurol 173:53-80.

Jones EG, Wise SP, Coulter JD (1979) Differential thalamic relationship of sensory-motor and parietal cortical fields in monkeys. J Comp Neurol 183:833-882.

Kawaguchi Y (1993) Physiological, morphological, and histochemical characterization of three classes of interneurons in rat neostriatum. J Neurosci 13:4908-4923.

Kawaguchi Y, Wilson CJ, Augood SJ, Emson PC (1995) Striatal interneurones: chemical, physiological and morphological characterization. Trends Neurosci 18:527-535.

Kimura M (1990) Behaviourally contingent property of movementrelated activity of the primate putamen. J Neurophysiol 61:1277-1296.

Kita H, Kosaka T, Heizmann CW (1990) Parvalbumin-immunoreactive neurons in the rat neostriatum: a light and electron microscopic study. Brain Res 536:1-15.

Konradi C, Kobierski LA, Nguyen TV, Heckers S, Hyman SE (1993) The cAMP-response-element-binding protein interacts, but Fos protein does not interact, with the proenkephalin enhancer in rat striatum. Proc Natl Acad Sci USA 90:7005-7009.

Korsching S (1993) The neurotrophic factor concept: a reexamination. J Neurosci 13:2739-2748.

Liste I, Rozas G, Guerra MJ, Labandeira-Garcia JL (1995) Cortical stimulation induces Fos expression in striatal neurons via NMDA glutamate and dopamine receptors. Brain Res 700:1-12.

Liu F-C, Graybiel AM (1996) Spatiotemporal dynamics of CREB phosphorylation: transient vs. sustained phosphorylation in the developing striatum. Neuron, in press.

Maneuf YP, Crossman AR, Brotchie JM (1996) Modulation of GABA transmission in the globus pallidus by the synthetic cannabinoid WIN 55,212-2. Synapse 22:382-385.

Mesulam M-M (1978) Tetramethyl benzidine for horseradish peroxidase neurohistochemistry: a non-carcinogenic blue reaction product with superior sensitivity for visualizing neural afferents and efferents. J Histochem Cytochem 26:106-117.

Parent A, Hazrati LN (1995) Functional anatomy of the basal ganglia. II. The place of subthalamic nucleus and external pallidum in basal ganglia circuitry. Brain Res Rev 20:128-154.
Parthasarathy HB (1996) Modular organization of the primate corticostriatal system. PhD thesis, Massachusetts Institute of Technology.

Parthasarathy HB, Schall JD, Graybiel AM (1992) Distributed but convergent ordering of striatal projections: the frontal eye field and the supplementary eye field in the monkey. J Neurosci 12:4468-4488.

Parthasarathy HB, Berretta S, Graybiel AM (1994) Cortical stimulation induces selective patterns of Jun B expression in the striatum. Soc Neurosci Abstr 20:987.

Peng ZC, Chen S, Fusco M, Vantini G, Bentivoglio M (1993) Fos induction by nerve growth factor in the adult rat brain. Brain Res 632:57-67.

Penny GR, Afsharpour S, Kitai ST (1986) The GAD, Leu-enkephalin, met-enkephalin, and substance P-immunoreactive neurons in the neostriatum of the rat and cat: evidence for partial population overlap. Neuroscience 17:1011-1045.

Plenz D, Aertsen A (1994) The basal ganglia: "minimal coherence detection" in cortical activity distributions. In: The basal ganglia. IV. New ideas and data on structure and function (Percheron G, McKenzie JS, Feger J, eds), pp 579-588. New York: Plenum.

Raichle ME (1994) Images of the mind: studies with modern imaging techniques. Annu Rev Psychol 45:333-356.

Rajakumar N, Elisevich K, Flumerfelt BA (1993) Biotinylated dextran: a versatile anterograde and retrograde neuronal tracer. Brain Res 607:47-53.

Reiner A, Veenman CL, Honig MG (1993) Anterograde tracing using biotinylated dextran amine. Neurosci Protocols 93-050-14-01.

Sagar SM, Sharp FR, Curran T (1988) Expression of c-fos protein in brain: metabolic mapping at the cellular level. Science 240:1328-1331.

Schultz R, Romo R (1992) Role of the primate basal ganglia and frontal cortex in the internal generation of movements. I. Preparatory activity in the anterior striatum. Exp Brain Res 91:363-384.

Sheng M, Greenberg ME (1990) The regulation and function of c-fos and other immediate early genes in the nervous system. Neuron 4:477-485.

Shivers BD, Harlan RE, Romano GJ, Howells RD, Pfaff DW (1986) Cellular localization of preenkephalin mRNA in rat brain: gene expression in the caudate-putamen and cerebellar cortex. Proc Natl Acad Sci USA 83:6221-6225.

Sokoloff L, Reivich M, Kennedy C, Des Rosiers MH, Patlak CS, Pettigrew KD, Sakurada O, Shinohara M (1977) The $\left[{ }^{14} \mathrm{C}\right]$ deoxyglucose method for the measurement of local cerebral glucose utilization: theory, procedure, and normal values in the conscious and anesthetized albino rat. J Neurochem 28:897-916.

Sonnenberg JL, Rauscher FJ, Morgan JI, Curran T (1989) Regulation of proenkephalin by Fos and Jun. Science 246:1622-1625.

Strick PL, Dum RP, Picard N (1995) Macro-organization of the circuits connecting the basal ganglia with the cortical motor areas. In: Models of information processing in the basal ganglia (Houk JC, Davis JL, Beiser DG, eds). Cambridge, MA: MIT.

Uhl GR, Navia B, Douglas J (1988) Differential expression of preproenkephalin and preprodynorphin mRNAs in striatal neurons: high levels of preproenkephalin expression depend on cerebral cortical afferents. J Neurosci 8:4755-4764.

Veenman CL, Reiner A, Honig MG (1992) Biotinylated dextran amine as an anterograde tracer for single-and double-labeling studies. J Neurosci Methods 41:239-254.

Vincent SR, Johansson O, Hökfelt T, Skirboll L, Elde RP, Terenius L, Kimmel J, Goldstein M (1983) NADPH-diaphorase: a selective histochemical marker for striatal neurons containing both somatostatin and avian pancreatic polypeptide (APP)-like immunoreactivities. J Comp Neurol 217:252-263.

Wan XST, Liang F, Moret V, Wiesendanger M, Rouiller EM (1992) Mapping of the motor pathways in rats: c-fos induction by intracortical microstimulation of the motor cortex correlated with efferent connectivity of the site of cortical stimulation. Neuroscience 49:749-761.

Wilson CJ (1995) The contribution of cortical neurons to the firing pattern of striatal spiny neurons. In: Models of information processing in the basal ganglia (Houk JC, Davis JL, Beiser DG, eds), pp 29-50. Cambridge, MA: MIT.

Yeterian EH, Van Hoesen GW (1978) Cortico-striatal projections in the rhesus monkey: the organization of certain cortico-caudate connections. Brain Res 139:43-63. 